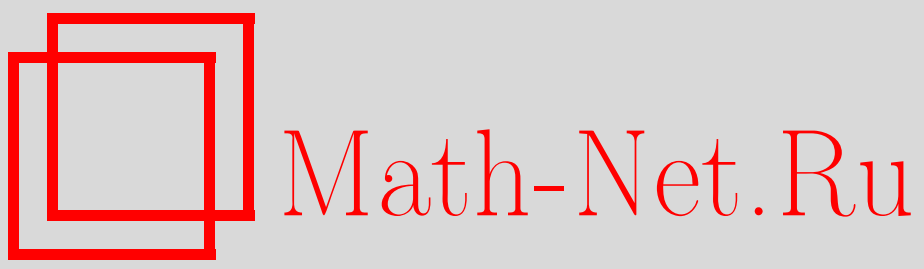

М. Энно, С. Трусарт, Асимптотическая структура гравитации в пространственной бесконечности в четырехмерном пространстве-времени, Труды МИAH, 2020, том 309, 141-164

DOI: https://doi.org/10.4213/tm4086

Использование Общероссийского математического портала Math-Net.Ru подразумевает, что вы прочитали и согласны с пользовательским соглашением http: //www . mathnet.ru/rus/agreement

Параметры загрузки:

IP: 54.84 .234 .179

26 апреля 2023 г., 17:37:26 
УДК $517.958:[531.123 .6+521.12+523.11]$

\title{
Асимптотическая структура гравитации в пространственной бесконечности в четырехмерном пространстве-времени*,**
}

\author{
М. Энно ${ }^{a, 6, \theta}$, С. Трусарт ${ }^{2}$
}

Поступило 09.04.2019; после доработки 22.08.2019; принято к публикации 15.05.2020

\begin{abstract}
Представлен обзор результатов, полученных авторами по асимптотической структуре гравитации на пространственной бесконечности в четырех пространственно-временны́х измерениях. Конечность действия и интегрируемость асимптотических генераторов лоренцева буста являются ключевыми критериями, выполнение которых обеспечивается соответствующими граничными условиями. Эти условия представляют собой "твистованные условия четности” и выражают тот факт, что в ведущем порядке асимптотики при антиподальном отображении сферы поля подчиняются строгим условиям четности с точностью до некоторого несобственного калибровочного преобразования. Показано, что асимптотические симметрии образуют бесконечномерную группу БондиМетцнера-Сакса, которая обладает нетривиальным действием. Найдены заряды и их алгебра. Изложение имеет целью самодостаточное представление материала и обладает педагогической составляющей.
\end{abstract}

DOI: https://doi.org/10.4213/tm4086

\section{1. ВВЕДЕНИЕ}

Настоящая статья посвящена нашему коллеге и другу Андрею Алексеевичу Славнову по случаю его 80-летия. Его способность проникнуть в суть симметрии и интерес к инфракрасной структуре калибровочных теорий (см., например, [28]) остаются для нас бесценными источниками вдохновения.

Мы представляем обзор наших недавних работ [18-22] (особенно [20]), посвященных асимптотической структуре гравитации и калибровочных теорий в пространственной бесконечности. Исследуется асимптотическая динамика полей на пространственноподобных гиперповерхностях, которые приближают пространственные гиперплоскости (гиперплоскости Коши) в бесконечности. В качестве основного инструмента используется гамильтонов формализм общей теории относительности $[12,1]$; корни нашего исследования лежат в пионерской работе [25]. Наша

\footnotetext{
* Статья представлена на английском языке. Оригинал опубликован в англоязычной версии журнала в режиме свободного доступа на условиях лицензии Creative Commons Attribution 4.0 International License (http://creativecommons.org/licenses/by/4.0/): Henneaux M., Troessaert C. The asymptotic structure of gravity at spatial infinity in four spacetime dimensions // Proc. Steklov Inst. Math. 2020. V. 309.

** Работа выполнена при частичной финансовой поддержке Европейского исследовательского совета (ЕRC Advanced Grant "High-Spin-Grav") и Национального фонда научных исследований Бельгии (FNRS, соглашение IISN 4.4503.15).

${ }^{a}$ Université Libre de Bruxelles, Brussels, Belgium.

${ }^{\sigma}$ International Solvay Institutes, Brussels, Belgium.

${ }^{8}$ On leave of absence from Collège de France, Paris, France.

${ }^{2}$ Max-Planck-Institut für Gravitationsphysik (Albert-Einstein-Institut), Potsdam, Germany.

E-mail: henneaux@ulb.ac.be (М. Энно), cedric.troessaert@hers.be (С. Трусарт).
}

(c) М. Энно, С. Трусарт, 2020 
главная цель - понять, как в пространственной бесконечности возникает симметрия БондиМетцнера-Сакса (БМС), изначально обнаруженная в светоподобной бесконечности $[7,26,27]$.

Имеются различные причины для изучения асимптотической структуры гравитации в пространственной бесконечности на пространственноподобных гиперповерхностях. Перечислим их кратко.

1. Существование светоподобной бесконечности является весьма непростой динамической проблемой (какие условия для данных Коши приводят к светоподобной бесконечности с достаточными свойствами гладкости?), и поэтому представляется разумным исследовать симметрии теории в контексте, который не зависит от решения этой проблемы. Это дает более твердую основу для исследования, а также отделяет существование симметрии БМС как симметрии теории от гравитационного излучения, которое, как известно, потенциально может разрушить обычные требования гладкости, наложенные в светоподобной бесконечности [11].

2. В предыдущих исследованиях гамильтоновой структуры на пространственной бесконечности группа БМС не отождествлялась с группой физических симметрий, в результате чего возникала несогласованность результатов на пространственной и светоподобной бесконечностях. Нам представляется важным устранить эти возникшие ранее противоречия.

3. Наконец, квантовую формулировку теории и такие вопросы, как тождества Уорда или одевание физических состояний, можно естественным образом исследовать на гиперповерхностях Коши.

Вопрос о том, как сформулировать непротиворечивые граничные условия для заданных законов динамики, не имеет однозначного ответа. В работах $[18,20]$ мы предложили два набора граничных условий для гравитации на пространственной бесконечности, которые инвариантны относительно группы БМС и приводят к корректно определенному и нетривиальному каноническому действию этой группы. Оба набора допустимы с гамильтоновой точки зрения, но мы полагаем, что некоторые преимущества имеют граничные условия из работы [20]:

(i) они допускают решения Тауба-Ньюмена-Унти-Тамбурино [24, 10];

(ii) они не приводят к логарифмическим расходимостям кривизны при стремлении к светоподобной бесконечности (хотя в общем случае присутствуют полилогарифмические члены в следующем за ведущим порядке) [30, 18-20].

Как было показано в работе [25], стандартное условие убывания пространственной метрики и ее сопряженного импульса, обычно предполагаемое на пространственной бесконечности, не приводит к корректно определенной канонической формулировке теории. Среди прочих трудностей отметим тот факт, что заряды однородной группы Лоренца в общем случае расходятся; следовательно, необходимо сделать это убывание более быстрым. Авторы работы [25] преодолели данную проблему путем наложения на ведущие компоненты метрики и ее импульса условий четности: при антиподальном отображении $x^{i} \rightarrow-x^{i}$ ведущие компоненты метрики четные, а ведущие компоненты их импульсов нечетные (в асимптотически декартовой системе координат). Из этих условий четности следует, что и электрические, и магнитные компоненты тензора Вейля имеют четные ведущие члены [20].

Далее, метрические компоненты и их сопряженные импульсы не инвариантны относительно диффеоморфизмов. Основная идея из [20] заключается в том, чтобы наложить условия четности, но твистованные. А именно, мы не требуем, чтобы члены компонент метрики (и их канонических импульсов) были строго четными (соответственно строго нечетными) в ведущем порядке, а требуем только четности (соответственно нечетности) с точностью до диффеоморфизма, который не обязан иметь определенную четность ${ }^{1}$.

\footnotetext{
1Это эквивалентно точным условиям четности для ведущих порядков компонент (инвариантного) тензора Вейля, а именно: они должны быть четными в декартовых координатах, если наложить дополнительные
} 
Если бы необходимые для обеспечения точных условий четности диффеоморфизмы представляли собой преобразования, не меняющие физического состояния системы, то можно было бы наложить точные условия четности на неинвариантные метрические компоненты и их импульсы. Однако, как было четко объяснено в глубокой работе [6], нужно различать "собственные" и "несобственные" калибровочные преобразования. В то время как первые имеют нулевые заряды и соответствуют избыточности в описании системы, вторые имеют ненулевые заряды и являются физическими преобразованиями, которые не могут быть фиксированы калибровкой. По этой причине они должны быть сохранены в асимптотике полей, и было бы неверно положить их равными нулю.

Такое идейно простое и, возможно, даже очевидное обобщение работы [25] (условия четности содержат "твист", заданный несобственным диффеоморфизмом) позволяет полностью согласовать анализ симметрии на пространственной и светоподобной бесконечностях. В частности, можно доказать, что на пространственной и светоподобной бесконечностях действует одна и та же группа БМС, но представленная с помощью различных параметризаций, которые можно явным образом связать друг с другом $[30,20]$. Однако чисто технически получить гамильтонов формализм с этими более общими условиями четности совсем напрямую нельзя, поскольку гамильтоновы переменные не инвариантны относительно диффеоморфизмов. Соответствующая необходимая работа была проделана в [20].

Цель настоящей статьи - дать систематический обзор результатов работы [20], имеющий самодостаточный характер, с определенным акцентом на педагогической стороне изложения. Наш обзор проливает свет на некоторые особенности вывода гамильтонова формализма.

\section{2. ДЕЙСТВИЕ И ОБОЗНАЧЕНИЯ}

Поскольку всюду далее мы используем гамильтонову формулировку общей теории относительности, кратко напомним ее основные положения. Гамильтоново действие чистой гравитации в четырехмерном пространстве-времени записывается как

$$
\begin{gathered}
S\left[g_{i j}, \pi^{i j}, N, N^{i}\right]=\int d t\left\{\int d^{3} x\left(\pi^{i j} \partial_{t} g_{i j}-N^{i} \mathcal{H}_{i}^{\text {grav }}-N \mathcal{H}^{\text {grav }}\right)-B_{\infty}\right\}, \\
\mathcal{H}^{\text {grav }}=-\sqrt{g} R+\frac{1}{\sqrt{g}}\left(\pi^{i j} \pi_{i j}-\frac{1}{2} \pi^{2}\right), \quad \mathcal{H}_{i}^{\text {grav }}=-2 \nabla_{j} \pi_{i}^{j},
\end{gathered}
$$

где $g_{i j}$ - трехмерная метрика на (пространственноподобных) сечениях постоянного времени, $g$ - ее определитель, $\pi^{i j}$ - сопряженный импульс, $N-$ функция хода, $N^{i}-$ функции сдвига $[12,1]$. Пространственная ковариантная производная по метрике $g_{i j}$ обозначается через $\nabla_{i}$. Скалярная кривизна метрики $g_{i j}$ есть $R$. В формуле для действия $B_{\infty}-$ граничный член на 2-сфере в пространственной бесконечности, который зависит от асимптотических значений функций хода и сдвига (см. соотношение (6.11) ниже). Варьируя действие по функциям хода и сдвига, приходим к условиям связи $\mathcal{H}^{\text {grav }} \approx 0$ и $\mathcal{H}_{i}^{\text {grav }} \approx 0$ для “гамильтониана" и "импульса". Таким образом, функции хода и сдвига работают как множители Лагранжа для связей.

Нас интересует пространство-время, которое при больших расстояниях приближается пространством-временем Минковского. В следующем разделе мы приводим точные скорости убывания различных полей. Начиная с этого момента обозначение $x^{i}(i=1,2,3)$ относится к асимптотическим декартовым координатам (кроме приложения $\mathrm{A}$ ). Асимптотические полярные координаты обозначаются через $\left(r, x^{A}\right)$, где $r^{2}=\sum_{i}\left(x^{i}\right)^{2}$ и $x^{A}(A=1,2)$ - координаты на единичной сфере (называемые углами). Плоская метрика, к которой на бесконечности

условия на некоторые БМС-инвариантные асимптотические поля, характеризующие структуру в пространственной бесконечности (см. [20, Appendix A.2] и заключительный раздел настоящей работы).

ТРУДЫ МАТЕМАТИЧЕСКОГО ИНСТИТУТА ИМ. В.А. СТЕКЛОВА, 2020, т. 309 
стремится пространственная метрика, имеет вид $\delta_{i j} d x^{i} d x^{j}=d r^{2}+r^{2} \bar{\gamma}_{A B} d x^{A} d x^{B}$, где $\bar{\gamma}_{A B}-$ метрика на единичной сфере. Большие расстояния соответствуют $r \rightarrow \infty$. Функции на единичной сфере обозначаются через $\bar{f}\left(x^{A}\right)$ или $\bar{f}\left(\mathbf{n}^{i}\right)$, где $\mathbf{n}^{i}$ - единичная нормаль к сфере. Величины с чертой сверху всегда определены на двумерной сфере и зависят только от углов (но в тексте также встречаются величины на 2-сфере, обозначения которых не содержат черты сверху; это будет ясно из контекста). Таким образом, например, $\bar{v}^{A}$ - это вектор на 2-сфере. Индексы $A, B, \ldots$ полей с чертой поднимаются и опускаются с помощью метрики $\bar{\gamma}_{A B}$ и обратной к ней $\bar{\gamma}^{A B}$, зависимость от $r$ при этом не появляется. Так, $\bar{v}_{A}=\bar{\gamma}_{A B} \bar{v}^{B}, \bar{v}^{A}=\bar{\gamma}^{A B} \bar{v}_{B}$ и т.д.

Преобразование четности - это преобразование $x^{k} \rightarrow-x^{k}$, которое индуцирует антиподальное отображение сферы $\mathbf{n}^{k} \rightarrow-\mathbf{n}^{k}$ вместе с $r \rightarrow r$. Это преобразование имеет корректно определенную асимптотику. В терминах углов $x^{A}$ антиподальное отображение сферы символически записывается как $x^{A} \rightarrow-x^{A}$ (на самом деле это $\theta \rightarrow \pi-\theta$ и $\varphi \rightarrow \varphi+\pi$ в обычных угловых переменных $(\theta, \varphi)$, где $\left.\bar{\gamma}_{A B} d x^{A} d x^{B}=(d \theta)^{2}+\sin ^{2} \theta(d \varphi)^{2}\right)$.

Геометрия плоского пространства. Поскольку это важно для рассмотрения ведущего порядка в пределе $r \rightarrow \infty$, полезно вспомнить несколько элементарных свойств сфер с центром в начале координат в евклидовой геометрии с индуцированной метрикой $\stackrel{\gamma}{A B}=r^{2} \bar{\gamma}_{A B}$.

Компоненты единичной нормали $\mathbf{n}$ к сфере задаются как $n^{i}=\partial x^{i} / \partial r$. Введем систему координат $\left\{n^{i} \equiv \partial x^{i} / \partial r=x^{i} / r, e_{A}^{i} \equiv \partial x^{i} / \partial x^{A}\right\}$, касательную к сферическим координатным линиям. Дуальная система есть $\left\{n_{i}=\partial r / \partial x^{i}, e^{A}{ }_{i}=\partial x^{A} / \partial x^{i}\right\}$. Имеем

$$
\begin{aligned}
& \delta_{i j} n^{i} n^{j}=1, \quad \delta_{i j} n^{i} e^{j}{ }_{A}=0, \quad \delta_{i j} e_{A}^{i} e_{B}^{j}=r^{2} \bar{\gamma}_{A B}, \\
& \delta^{i j} n_{i} n_{j}=1, \quad \delta^{i j} n_{i} e^{A}{ }_{j}=0, \quad \delta^{i j} e^{A}{ }_{i} e^{B}{ }_{j}=\frac{1}{r^{2}} \bar{\gamma}^{A B} .
\end{aligned}
$$

Нетрудно получить соотношения

$$
\partial_{j} n^{i}=\frac{1}{r}\left(\delta_{j}^{i}-n^{i} n_{j}\right), \quad \partial_{i} e_{k}^{A}=-\frac{1}{r}\left(n_{k} e_{i}^{A}+n_{i} e_{k}^{A}\right)-{ }^{(2)} \bar{\Gamma}_{B C}^{A} e_{i}^{B} e_{k}^{C},
$$

где ${ }^{(2)} \bar{\Gamma}_{B C}^{A}-$ символ Кристоффеля для метрики $\bar{\gamma}_{A B}$.

Обозначим ковариантную производную по трехмерной плоской метрике через $\stackrel{\circ}{\nabla}_{i}$. В общем случае символ ${ }^{\circ}$ над величиной относится к евклидову пространству. Двумерные ковариантные производные по метрикам $\stackrel{\gamma}{\gamma}_{A B}$ и $\bar{\gamma}_{A B}$ обозначаются через $\dot{\circ}_{A}$ и $\bar{D}_{A}$ соответственно ${ }^{2}$. Соответствующие кривизны ${ }^{(2)} \stackrel{\circ}{R}$ и ${ }^{(2)} \bar{R}$ суть ${ }^{(2)} \stackrel{\circ}{R}_{A B}={ }^{(2)} \bar{R}_{A B}=\bar{\gamma}_{A B},{ }^{(2)} \stackrel{\circ}{R}=2 / r^{2},{ }^{(2)} \bar{R}=2$.

Внешняя кривизна $\stackrel{\circ}{K}_{A B}$ сферы радиуса $r$ в евклидовом пространстве определяется как $e_{A}^{i} \stackrel{\circ}{\nabla}_{i} n^{j}=-\stackrel{\circ}{K}_{A}^{B} e_{B}^{j}$. Отсюда $\stackrel{\circ}{K}_{A B} \equiv \stackrel{\circ}{\gamma}_{B C} \stackrel{\circ}{K}_{A}^{C}=-r \bar{\gamma}_{A B}, \stackrel{\circ}{K}_{A}^{B}=-\delta_{A}{ }^{B} / r$. Внешняя кривизна удовлетворяет уравнениям Гаусса-Кодацци $\stackrel{\circ}{K}_{A}^{B} \stackrel{\circ}{K}_{B}^{A}-\stackrel{\circ}{K}^{2}+{ }^{(2)} \stackrel{\circ}{R}=0, \partial_{A} \stackrel{\circ}{K}-\stackrel{\circ}{D}_{B} \stackrel{\circ}{K}^{B}{ }_{A}=0$.

\section{3. ГРАНИЧНЫЕ УСЛОВИЯ}

Прежде всего примем в качестве асимптотического поведения метрики и ее сопряженного импульса следующее убывание в декартовых координатах:

$$
g_{i j}=\delta_{i j}+O\left(r^{-1}\right), \quad \pi^{i j}=O\left(r^{-2}\right) .
$$

Такое асимптотическое убывание необходимо усилить. В самом деле, если не накладывать дополнительные условия на ведущий порядок $O\left(r^{-1}\right)$ метрики и порядок $O\left(r^{-2}\right)$ сопряженного импульса, то возникают две проблемы.

\footnotetext{
${ }^{2}$ На самом деле $\stackrel{\circ}{D}_{A}=\bar{D}_{A}$, поскольку две метрики связаны преобразованием масштаба. Однако в асимптотически плоской геометрии метрика $\gamma_{A B}$ на сферах с постоянным радиусом $r$ задается формулой $\gamma_{A B}=\stackrel{\circ}{\gamma}_{A B}+\left(\right.$ следующие за ведущим члены), поэтому ковариантная производная $D_{A}$ относительно $\gamma_{A B}$ отличается от $\bar{D}_{A}$ в порядках, следующих за ведущим. Метрика $\bar{\gamma}_{A B}$ по-прежнему равна $\lim _{r \rightarrow \infty} \gamma_{A B} / r^{2}$, тем самым задается поведение метрики $\gamma_{A B}$ в ведущем порядке.
} 
- Во-первых, кинетический член в действии расходится, поскольку в лидирующем порядке он ведет себя как $1 / r^{3}$, что, вообще говоря, приводит к логарифмической расходимости при интегрировании по всему пространству (которое включает в себя $\int r^{2} d r$, что дает $\left.\int d r / r\right)$. Конечность действия - это важное физическое требование.

- Во вторых, в асимптотике бусты имеют неинтегрируемые генераторы, т.е. не являются каноническими преобразованиями (см. п. 6.4). Поскольку асимптотически плоское пространство должно быть инвариантным относительно бустов, это опять же нежелательно.

Один из способов решения первой проблемы мог бы заключаться в наложении требования противоположных свойств четности для ведущих членов метрики и ее сопряженного импульса при антиподальном отображении, а именно [25]

$$
h_{i j} \equiv g_{i j}-\delta_{i j}=\frac{\bar{h}_{i j}\left(\mathbf{n}^{k}\right)}{r}+O\left(\frac{1}{r^{2}}\right), \quad \bar{h}_{i j}\left(-\mathbf{n}^{k}\right)=\bar{h}_{i j}\left(\mathbf{n}^{k}\right)
$$

и

$$
\pi^{i j}=\frac{\bar{\pi}^{i j}\left(\mathbf{n}^{k}\right)}{r^{2}}+O\left(\frac{1}{r^{3}}\right), \quad \bar{\pi}^{i j}\left(-\mathbf{n}^{k}\right)=-\bar{\pi}^{i j}\left(\mathbf{n}^{k}\right)
$$

Ведущий порядок в $\pi^{i j} \partial_{t} g_{i j}$ тогда имеет вид $f\left(\mathbf{n}^{k}\right) / r^{3}$, где $f\left(\mathbf{n}^{k}\right)$ - нечетная функция на сфере, в итоге логарифмическая расходимость типа $\int d r / r$ умножается на равный нулю интеграл $\int f(\theta, \varphi) \sin \theta d \theta d \varphi$.

Как подчеркивалось в работе [20], эти точные условия четности нужно ослабить (без потери полезного свойства конечности кинетического члена в действии), поскольку для получения системы координат, в которой сохраняются условия четности, возможно, придется выполнить "несобственное калибровочное преобразование" [6], а такая фиксация калибровки недопустима.

В работе [20] мы предложили заменить точные условия четности на условия четности с "твистом", который задается несобственным калибровочным преобразованием ${ }^{3}$. Точнее, в $\bar{h}_{i j}\left(\mathbf{n}^{k}\right)$ допускаются и четная $\left(\bar{h}_{i j}\right)^{\text {even }}\left(\mathbf{n}^{k}\right)$, и нечетная $\left(\bar{h}_{i j}\right)^{\text {odd }}\left(\mathbf{n}^{k}\right)$ компоненты, но если четная компонента произвольна, то нечетная компонента должна быть диффеоморфизмом специального вида (стремиться к константе на бесконечности для сохранения асимптотического убывания со скоростью $\left.r^{-1}\right)$. В ведущем порядке и в плоской фоновой метрике изменение поля $h_{i j}$ под действием диффеоморфизма принимает линейный вид $\partial_{i} \zeta_{j}+\partial_{j} \zeta_{i}$, где $\zeta^{i}$ имеет порядок единицы (т.е. это функция, зависящая только от углов). Таким образом, мы требуем выполнения равенств

$$
\begin{aligned}
& h_{i j} \equiv g_{i j}-\delta_{i j}=U_{i j}+j_{i j}, \\
& U_{i j}=\partial_{i} \zeta_{j}+\partial_{j} \zeta_{i}=O\left(\frac{1}{r}\right), \quad \zeta^{i}=\zeta^{i}\left(\mathbf{n}^{k}\right)=O(1), \quad \zeta^{i}\left(-\mathbf{n}^{k}\right)=\zeta^{i}\left(\mathbf{n}^{k}\right), \\
& j_{i j}=\frac{\left(\bar{h}_{i j}\right)^{\operatorname{even}}\left(\mathbf{n}^{k}\right)}{r}+O\left(\frac{1}{r^{2}}\right), \quad\left(\bar{h}_{i j}\right)^{\text {even }}\left(-\mathbf{n}^{k}\right)=\left(\bar{h}_{i j}\right)^{\text {even }}\left(\mathbf{n}^{k}\right)
\end{aligned}
$$

для некоторых $\zeta^{i}$, которые можно считать четными функциями, поскольку нечетные компоненты могут быть поглощены за счет переопределения $\left(\bar{h}_{i j}\right)^{\text {even }}$. Поскольку $\zeta^{i}$ имеет порядок единицы (более низкие порядки поглощаются за счет переопределения низших порядков в

\footnotetext{
${ }^{3}$ Несобственные калибровочные преобразования в гамильтоновой форме задаются соотношениями (5.1) и (5.2). Функция $\zeta$ и вектор $\zeta^{i}$, имеющие порядок единицы, в асимптотике представляют собой нормальную и касательную компоненты этих преобразований (они не содержат ни буста, ни вращения). В ведущем порядке имеют значение только часть $\mathcal{L}_{\xi^{m}} g_{i j}$ в $\delta g_{i j}$ и часть $g^{1 / 2}\left(\zeta^{\mid i j}-g^{i j} \zeta^{\mid m}{ }_{\mid m}\right)$ импульса $\pi^{i j}$.
} 
функциях $j_{i j}$, не ограниченных условиями четности), отсюда получаем

$$
U_{i j}=\frac{\left(\bar{h}_{i j}\right)^{\text {odd }}\left(\mathbf{n}^{k}\right)}{r} \quad \Leftrightarrow \quad\left(\bar{h}_{i j}\right)^{\text {odd }}\left(\mathbf{n}^{k}\right)=r U_{i j}
$$

где

$$
U_{i j}=e_{i}^{A} \partial_{A} \zeta_{j}+e_{j}^{A} \partial_{A} \zeta_{i} .
$$

Аналогичным образом мы допускаем как нечетные $\left(\bar{\pi}^{i j}\right)^{\text {odd }}\left(\mathbf{n}^{k}\right)$, так и четные $\left(\bar{\pi}^{i j}\right)^{\text {even }}\left(\mathbf{n}^{k}\right)$ компоненты функции $\bar{\pi}^{i j}$. Нечетная составляющая не ограничена никакими условиями, а четная должна получаться из преобразования импульса $\pi^{i j}$ под действием диффеоморфизма (стремящегося к константе для сохранения асимптотического убывания со скоростью $r^{-2}$ ). В ведущем порядке на $\pi^{i j}$ действуют только нормальные диффеоморфизмы. Обозначим нормальную компоненту диффеоморфизма через $V$, она имеет порядок единицы. Тогда в ведущем порядке преобразование принимает вид $\partial^{i} \partial^{j} V-\delta^{i j} \stackrel{\Delta}{\Delta} V$. Здесь $\stackrel{\circ}{\Delta} \stackrel{\circ}{\nabla}^{i} \stackrel{\circ}{\nabla}_{i}-$ лапласиан в плоской метрике. Итак, возьмем

$$
\begin{aligned}
& \pi^{i j}=V^{i j}+p^{i j}, \\
& V^{i j}=\partial^{i} \partial^{j} V-\delta^{i j} \stackrel{\circ}{V}, \quad V=V\left(\mathbf{n}^{k}\right)=O(1), \quad V\left(-\mathbf{n}^{k}\right)=V\left(\mathbf{n}^{k}\right), \\
& p^{i j}=\frac{\left(\bar{\pi}^{i j}\right)^{\text {odd }}\left(\mathbf{n}^{k}\right)}{r^{2}}+O\left(\frac{1}{r^{3}}\right), \quad\left(\bar{\pi}^{i j}\right)^{\text {odd }}\left(-\mathbf{n}^{k}\right)=-\left(\bar{\pi}^{i j}\right)^{\text {odd }}\left(\mathbf{n}^{k}\right)
\end{aligned}
$$

при некоторой $V$, которую можно считать четной, поскольку нечетные компоненты могут быть поглощены путем переопределения $\left(\bar{\pi}^{i j}\right)^{\text {odd }}$. Так как $V$ имеет порядок единицы (более низкие порядки поглощаются за счет переопределения низших порядков в функциях $p^{i j}$, не ограниченных условиями четности), отсюда получаем

$$
V^{i j}=\frac{\left(\bar{\pi}^{i j}\right)^{\mathrm{even}}\left(\mathbf{n}^{k}\right)}{r^{2}} \quad \Leftrightarrow \quad\left(\bar{\pi}^{i j}\right)^{\mathrm{even}}\left(\mathbf{n}^{k}\right)=r^{2} \delta^{i m} \delta^{j n} V_{m n},
$$

где

$$
\begin{aligned}
V_{m n} & =\partial_{m} \partial_{n} V-\delta_{m n} \stackrel{\circ}{V}= \\
& =-n_{m} n_{n} \frac{1}{r^{2}} \bar{\Delta} V-\frac{1}{r}\left(n_{m} e_{n}^{A}+n_{n} e_{m}^{A}\right) \bar{D}_{A} V+e_{m}^{A} e_{n}^{B}\left(\bar{D}_{A} \bar{D}_{B} V-\bar{\gamma}_{A B} \bar{\Delta} V\right)
\end{aligned}
$$

и $\bar{\Delta} \equiv \bar{\gamma}^{A B} \bar{D}_{A} \bar{D}_{B}-$ лапласиан на сфере (с метрикой $\left.\bar{\gamma}_{A B}\right)$.

Поскольку преобразования линеаризуются в бесконечности, конечные формы несобственных калибровочных преобразований $U_{i j}$ и $V^{i j}$ совпадают с их инфинитезимальными формами. Поэтому мы можем считать, что в приведенных выше соотношениях $\zeta^{i}$ и $V$ конечны, а не просто бесконечно малы.

Добавление к динамическим переменным этих несобственных компонент калибровочного преобразования не совсем тривиально. Оно усложняет вид зарядов и приводит к потере полезного свойства конечности симплектического члена в действии. Чтобы устранить расходимость симплектической формы, сохраняя свободу выполнения несобственных калибровочных преобразований, мы требуем выполнения условия связи для ведущих порядков. Связи $\mathcal{H}^{\text {grav }}$ и $\mathcal{H}_{i}^{\text {grav }}$ в случае общего убывания обычно ведут себя в декартовых координатах как $r^{-3}$. Мы требуем, чтобы их поведение было на два порядка ниже:

$$
\left.\mathcal{H}^{\text {grav }}=O\left(r^{-5}\right), \quad \mathcal{H}_{i}^{\text {grav }}=O\left(r^{-5}\right) \quad \text { (в декартовых координатах }\right) .
$$


На самом деле, для того чтобы симплектический член гамильтониана был конечным, требуется только асимптотика $\mathcal{H}^{\text {grav }}=O\left(r^{-4}\right)$ и $\mathcal{H}_{i}^{\text {grav }}=O\left(r^{-4}\right)$. Мы вводим более сильное условие (3.12), чтобы гарантировать, что объемные части генераторов буста и вращения, которые обычно ведут себя как $r \mathcal{H}^{\text {grav }}$ и $r \mathcal{H}_{i}^{\text {grav }}$, также являются сходящимися интегралами.

Полезно записать в явном виде условие, что в связях отсутствуют члены порядка $O\left(r^{-3}\right)$. Вводя обозначения $J_{i j}=\left(\bar{h}_{i j}\right)^{\text {even }} / r$ (первый член разложения для $j_{i j}$ ) и $P^{i j}=\left(\bar{\pi}^{i j}\right)^{\text {odd }} / r^{2}$ (первый член разложения для $\left.p^{i j}\right)$, получаем

$$
\partial^{i} \partial^{j} J_{i j}-\stackrel{\circ}{\Delta} J=0, \quad \partial_{i} P^{i j}=0 ;
$$

это условие не включает в себя несобственные компоненты калибровочных преобразований, поскольку для них автоматически выполняются соотношения $\partial^{i} \partial^{j} U_{i j}-\stackrel{\Delta}{\Delta}=0, \partial_{i} V^{i j}=0$. Ниже мы проверим, что это делает симплектическую форму конечной. Следующий член разложения связей можно найти в [18].

Требование, что условия связи на бесконечности выполнены с точностью на два порядка выше, чем это следует из убывания полей, является разумным (связи преобразуются друг в друга при преобразованиях Пуанкаре); оно не исключает никаких решений, для которых связи выполнены во всем пространстве, т.е. во всех порядках.

Для решения второй проблемы (отсутствия канонического генератора для бустов) потребуем, чтобы ведущий порядок смешанных радиально-угловых компонент метрики, которые ведут себя как $O(1)$, был на самом деле равен нулю, так что

$$
g_{r A} \equiv h_{r A}=O\left(\frac{1}{r}\right) .
$$

Отсюда следуют соотношения ${ }^{4}$

$$
\zeta_{i}=\partial_{i} \widetilde{U}, \quad \widetilde{U}=r \bar{U}, \quad \bar{U}=\bar{U}\left(\mathbf{n}^{k}\right)=O(1), \quad \bar{U}\left(-\mathbf{n}^{k}\right)=-\bar{U}\left(\mathbf{n}^{k}\right)
$$

для некоторой функции $\bar{U}$ угловых переменных, а также

$$
\bar{j}_{r A}=0 .
$$

Ниже мы явным образом проверим, что это решает вторую проблему.

Несобственные преобразования удовлетворяют интересным тождествам

$$
n^{n} U_{n m}=0
$$

и

$$
r^{2} n^{m} n^{n} V_{m n}=-\bar{\Delta} V=r \bar{D}^{A}\left(n^{m} e_{A}^{n} V_{m n}\right)=\bar{\gamma}^{A B} e_{A}^{m} e_{B}^{n} V_{m n}
$$

Для доказательства тождества (3.17) заметим, что для $\zeta_{i}$, заданной уравнением $\partial_{i}(r \bar{U})=n_{i} \bar{U}+$ $+r e_{i}^{A} \partial_{A} \bar{U}$, величина $U_{i j}$ принимает вид

$$
U_{i j}=r e_{i}^{A} e_{j}^{B} \bar{\Upsilon}_{A B}, \quad \bar{\Upsilon}_{A B}=2\left(\bar{U} \bar{\gamma}_{A B}+\bar{D}_{A} \bar{D}_{B} \bar{U}\right) .
$$

Из этого выражения также видно, что

$$
\partial_{k} U_{i j}=\left(-n_{k} e_{i}^{A} e_{j}^{B}-n_{i} e_{k}^{A} e_{j}^{B}-n_{j} e_{i}^{A} e_{k}^{B}\right) \bar{\Upsilon}_{A B}+r e_{i}^{A} e_{j}^{B} e_{k}^{C} \bar{D}_{C} \bar{\Upsilon}_{A B} .
$$

Условия (3.2)-(3.9), (3.12) и (3.15)-(3.16) составляют набор наших начальных условий.

\footnotetext{
${ }^{4}$ Условие $\stackrel{\circ}{\nabla}_{r} \zeta_{A}+\stackrel{\circ}{\nabla}_{A} \zeta_{r}=O(1 / r)$ эквивалентно точному уравнению $\stackrel{\circ}{\nabla}_{r} \zeta_{A}+\stackrel{\circ}{\nabla}_{A} \zeta_{r}=0$, поскольку $\zeta_{r}$ зависит только от углов, $\zeta_{r}=U\left(x^{A}\right)$, а $\zeta_{A}$ линейна по $r, \zeta_{A}=r \bar{\zeta}_{A}$ (предполагается, что $\zeta_{i}$ однородна, потому что ее члены низших порядков по $1 / r$ поглощаются функцией $j_{i j}$, следовательно, $\zeta_{i}$ зависит только от углов, но не от $r$ ). Непосредственным вычислением из условия $\dot{\nabla}_{r} \zeta_{A}+\stackrel{\circ}{\nabla}_{A} \zeta_{r}=0$ получаем $\bar{\zeta}_{A}=\partial_{A} U$. Таким образом, $\zeta_{r}=\partial_{r}(r U)$ и $\zeta_{A}=\partial_{A}(r U)$, т.е. $\zeta_{i}=\partial_{i}(r U)$.
} 


\section{4. КОНЕЧНОСТЬ СИМПЛЕКТИЧЕСКОЙ СТРУКТУРЫ}

Теперь проверим, что кинетический член $\int d^{3} x \pi^{i j} \partial_{t} g_{i j}$ в гамильтониане конечен, т.е. конечна симплектическая структура.

Имеются четыре члена: $\int d^{3} x V^{i j} \partial_{t} U_{i j}, \int d^{3} x p^{i j} \partial_{t} U_{i j}, \int d^{3} x V^{i j} \partial_{t} j_{i j}$ и $\int d^{3} x p^{i j} \partial_{t} j_{i j}$. Первый и последний из них конечны, так как в обоих случаях коэффициент при потенциально возможной логарифмической расходимости есть интеграл по сфере от нечетной функции, который равен нулю. Таким образом, требуется рассмотреть только два других члена.

Потенциально возможная расходимость в $\int d^{3} x p^{i j} \partial_{t} U_{i j}$ задается интегралом $\int d^{3} x P^{i j} \partial_{t} U_{i j}$, где $P^{i j}=\left(\bar{\pi}^{i j}\right)^{\text {odd }} / r^{2}$. Используя выражение для функции $U_{i j}$ и обозначение $\eta^{i}=\partial_{t} \zeta^{i}$, приведем последний интеграл к виду

$$
\int d^{3} x P^{i j} \partial_{t} U_{i j}=2 \int d^{3} x P^{i j} \partial_{i} \eta_{j}=2 \int d^{3} x \partial_{i}\left(P^{i j} \eta_{j}\right)-2 \int d^{3} x \partial_{i}\left(P^{i j}\right) \eta_{j} .
$$

Первое слагаемое в правой части можно преобразовать в поверхностный интеграл, конечность которого легко проверить. Второе слагаемое равно нулю в силу (3.13).

Аналогично возможная расходимость в $\int d^{3} x V^{i j} \partial_{t} j_{i j}$ дается интегралом $\int d^{3} x V^{i j} H_{i j}$, где $H_{i j}=\partial_{t}\left(\bar{h}_{i j}\right)^{\text {even }} / r$. Используя выражение для функции $V^{i j}$, приведем его к виду

$$
\begin{aligned}
\int d^{3} x V^{i j} H_{i j} & =2 \int d^{3} x\left(\partial^{i} \partial^{j} V-\delta^{i j} \stackrel{\circ}{V}\right) H_{i j}= \\
& =\text { Конечные поверхностные члены }+\int d^{3} x V\left(\partial^{i} \partial^{j} H_{i j}-\stackrel{\Delta}{\Delta} H\right) .
\end{aligned}
$$

Второе слагаемое равно нулю в силу (3.13). Таким образом, потенциально логарифмически расходящийся интеграл $\int d^{3} x V^{i j} \partial_{t} j_{i j}$ на самом деле также конечен.

Завершим данный раздел следующим замечанием. Наше рассуждение также показывает, что несобственный калибровочный член $\int d^{3} x V^{i j} \partial_{t} U_{i j}$ на самом деле является конечным без предположения об определенной четности функции $U_{i j}$ или $V_{i j}$. Например, это можно увидеть, выбрав $j_{i j}$ того же вида, что и $U_{i j}$ (но с $\zeta_{i}$ противоположной четности), а $p^{i j}$ взять того же вида, что и $V^{i j}$ (но с $V$ противоположной четности). Поскольку для этих членов в ведущем порядке выполняются условия связи, доказательство проходит без каких-либо других изменений.

\section{5. ИНВАРИАНТНОСТЬ ГРАНИЧНЫХ УСЛОВИЙ}

Когда параметризованная переменными $\left(\xi^{\perp} \equiv \xi, \xi^{i}\right)$ гиперповерхность постоянного времени подвергается деформации, канонические переменные преобразуются как $[12,1]$

$$
\begin{aligned}
\delta g_{i j}= & 2 \xi g^{-1 / 2}\left(\pi_{i j}-\frac{1}{2} g_{i j} \pi\right)+\mathcal{L}_{\xi} g_{i j}, \\
\delta \pi^{i j}= & -\xi g^{1 / 2}\left(R^{i j}-\frac{1}{2} g^{i j} R\right)+\frac{1}{2} \xi g^{-1 / 2}\left(\pi_{m n} \pi^{m n}-\frac{1}{2} \pi^{2}\right)- \\
& -2 \xi g^{-1 / 2}\left(\pi^{i m} \pi_{m}^{j}-\frac{1}{2} \pi^{i j} \pi\right)+g^{1 / 2}\left(\xi^{\mid i j}-g^{i j} \xi^{\mid m}\right)+\mathcal{L}_{\xi} \pi^{i j},
\end{aligned}
$$

где $\mathcal{L}_{\xi} g_{i j}$ и $\mathcal{L}_{\xi} \pi^{i j}$ - производные Ли функций $g_{i j}$ и $\pi^{i j}$ вдоль векторного поля $\xi^{i}$,

$$
\begin{aligned}
\mathcal{L}_{\xi} g_{i j} & =\xi_{i \mid j}+\xi_{j \mid i}, \\
\mathcal{L}_{\xi} \pi^{i j} & =\left(\pi^{i j} \xi^{m}\right)_{\mid m}-\xi^{i}{ }_{\mid m} \pi^{m j}-\xi^{j}{ }_{\mid m} \pi^{i m},
\end{aligned}
$$

а вертикальная черта $\mid$ означает ковариантную производную по полной метрике $g_{i j}$, т.е. ${ }_{i} \equiv \nabla_{i}$. 
Граничные условия инвариантны относительно деформаций гиперповерхности, которые асимптотически ведут себя как

$$
\begin{gathered}
\xi=b_{i} x^{i}+T(\mathbf{n})+C^{(b)}(\mathbf{n})+O\left(r^{-1}\right), \\
\xi^{i}=b_{j}^{i} x^{j}+I^{i}(\mathbf{n})+O\left(r^{-1}\right), \\
I_{i}(\mathbf{n})=W_{i}(\mathbf{n})+C_{i}^{(b)}(\mathbf{n}), \quad W_{i}(\mathbf{n})=\partial_{i}(r W(\mathbf{n})),
\end{gathered}
$$

где $b_{i}$ и $b_{i j}=-b_{j i}$ - произвольные постоянные, а $T(\mathbf{n})$ и $W(\mathbf{n})-$ произвольные функции на единичной сфере $\left(\mathbf{n}^{i}=x^{i} / r\right)$. Константы $b_{i}$ параметризуют лоренцевы бусты (в любой заданный момент времени соответствующий член $-b^{i} x^{0}$ в $\xi^{i}$ может быть поглощен величиной $a^{i}$ ). Антисимметричные константы $b_{i j}=-b_{j i}$ параметризуют пространственные вращения. Нулевые моды $a_{0}$ и $a_{0}^{i}$ в $T$ и $W^{i}$ представляют собой стандартные трансляции. В разложении $T\left(x^{A}\right)$ и $W\left(x^{B}\right)$ по сферическим гармоникам это соответствует выбору $T\left(x^{B}\right) \sim a_{0} Y_{0}^{0}$ и $W\left(x^{B}\right) \sim a_{0}^{m} Y_{m}^{1}$, но при этом допускаются высшие сферические гармоники.

"Поправочные члены" $C^{(b)}(\mathbf{n})$ и $C_{i}^{(b)}(\mathbf{n})$ появляются только тогда, когда преобразование включает в себя буст $\left(b_{i} \neq 0\right)$. Первый из этих членов необходим для интегрируемости зарядов буста, а второй - для выполнения условия $\bar{h}_{r A}=0$. Явный вид поправочных членов в сферических координатах приведен в п. 6.4. Они оба содержат поля, имеют порядок единицы и линейны по своим параметрам, так что $C^{(b)}(\mathbf{n})=0=C_{i}^{(b)}(\mathbf{n})$, если $b_{i}=0$.

Независимые параметры симметрий суть $b_{i}, b_{i j}, T(\mathbf{n})$ и $W(\mathbf{n})$. При этом граничные условия инвариантны относительно асимптотической алгебры, которая имеет алгебру Пуанкаре в качестве собственной подалгебры. Общие функции $T$ и $W$ описывают "зависящие от угла" трансляции, или "супертрансляции", и могут содержать компоненты с любой четностью. Нечетная часть функции $W$ приводит к сдвигу функции $U$, а четная часть изменяет $h_{i j}$. Аналогично четная часть функции $T$ приводит к сдвигу функции $V$, а нечетная часть изменяет $p^{i j}$. Точные правила преобразования функций $U$ и $V$ приведены в приложении Б. Оказывается, что только нечетная часть функции $W$ и четная часть функции $T$ определяют несобственные калибровочные преобразования. Прочие части представляют собой чистые калибровочные преобразования (см. следующий раздел).

\section{6. ПОВЕРХНОСТНЫЕ ЧЛЕНЫ И ГЕНЕРАТОРЫ ЗАРЯДОВ}

Генератор преобразований (5.5)-(5.7) имеет вид

$$
P_{\xi}^{\text {grav }}\left[g_{i j}, \pi^{i j}\right]=\int d^{3} x\left(\xi \mathcal{H}+\xi^{i} \mathcal{H}_{i}\right)+\mathcal{B}_{\xi}^{\text {grav }}\left[g_{i j}, \pi^{i j}\right],
$$

где граничный член $\mathcal{B}_{\xi}$ определяется с помощью метода из работы [25], т.е. он должен быть таким, чтобы внешняя производная $d_{V} P_{\xi}^{\text {grav }}$ от $P_{\xi}^{\text {grav }}$ в пространстве полей $\left(\mathrm{c}\left(\xi, \xi^{k}\right)\right.$, заданными в (5.5)-(5.7)) сводилась к интегралу по всему объему, включающему в себя только не подвергающиеся дифференцированию вариации полей $d_{V} g_{i j}, d_{V} \pi^{i j}$. Другими словами, $d_{V} \mathcal{B}_{\xi}^{\text {grav }}$ должна приводить к сокращению граничных членов, происходящих из $d_{V} \int d^{3} x\left(\xi \mathcal{H}+\xi^{i} \mathcal{H}_{i}\right)$ при интегрировании по частям, которое необходимо для приведения $d_{V} P_{\xi}^{\text {grav }}$ к подходящему объемному виду. При условии, что симплектическая форма $\Omega$ имеет стандартный вид объемного интеграла $\int d^{3} x d_{V} \pi^{i j} d_{V} g_{i j}$ без поверхностных членов, указанное выше требование эквивалентно тому, что преобразования (5.5)-(5.7) должны быть каноническими, т.е. оставлять инвариантной симплектическую форму, $d_{V} i_{\xi} \Omega=0$ (или, что равносильно, $i_{\xi} \Omega=-d_{V} P_{\xi}^{\text {grav }}$, см. [19]).

Явные вычисления приводят к тождеству [25]

$$
d_{V} \int d^{3} x\left(\xi \mathcal{H}+\xi^{i} \mathcal{H}_{i}\right)=\text { Необходимый объемный член }+\mathcal{K}_{\xi}\left[d_{V} g_{i j}, d_{V} \pi^{i j}\right],
$$


где

$$
\begin{aligned}
\mathcal{K}_{\xi}\left[d_{V} g_{i j}, d_{V} \pi^{i j}\right]= & -\oint G^{i j k l}\left(\xi\left(d_{V} g_{i j}\right)_{\mid k}-\xi_{, k} d_{V} g_{i j}\right) d^{2} S_{l}- \\
& -\oint\left(2 \xi_{k} d_{V} \pi^{k l}+\left(2 \xi^{k} \pi^{j l}-\xi^{l} \pi^{j k}\right) d_{V} g_{j k}\right) d^{2} S_{l} .
\end{aligned}
$$

В этом выражении $G^{i j k l}$ - суперметрика Девитта,

$$
G^{i j k l}=\sqrt{g}\left(\frac{1}{2}\left(g^{i k} g^{j l}+g^{i l} g^{j k}\right)-g^{i j} g^{k l}\right) .
$$

Поверхностный интеграл, возможно, расходится для бустов и пространственных вращений, которые растут линейно с ростом $r$.

6.1. Отсутствие расходимостей. Возможная расходимость для бустов имеет вид

$$
-\oint \stackrel{G}{G}^{i j k l}\left(\xi \stackrel{\circ}{\nabla}_{k}\left(d_{V} U_{i j}\right)-\xi_{, k} d_{V} U_{i j}\right) d^{2} S_{l}
$$

поскольку

(i) в ведущем порядке $G^{i j k l}$ совпадает с суперметрикой Девитта $G^{i j k l}$, если метрика плоская, и ковариантная производная $\left(d_{V} g_{i j}\right)_{\mid k}$ совпадает с ковариантной производной $\stackrel{\circ}{\nabla}_{k}\left(d_{V} g_{i j}\right)$ для плоской фоновой метрики;

(ii) в силу того, что для бустов переменная $\xi$ нечетная, вклад в интеграл вносит только ведущая нечетная компонента $U_{i j}$.

Четная компонента пропадает. Поскольку в ведущем порядке условия связи выполнены, а $\xi$ - вектор Киллинга, нечетная составляющая на самом деле тоже не вносит вклад: $U_{i j}$ также отсутствует, и дивергенция равна нулю. Это происходит потому, что подынтегральное выражение является точной 2-формой, которая при интегрировании дает нуль. Это можно проверить непосредственно, выразив ведущую компоненту связи и интеграл по 2-сфере в полярных координатах, как это сделано в п. 6.4 ниже, или заметив, что имеет место равенство

$$
\oint \stackrel{G}{G}^{i j k l}\left(\xi \partial_{k}\left(d_{V} U_{i j}\right)-\xi_{, k} d_{V} U_{i j}\right) d^{2} S_{l}=\int d^{3} x \partial_{l}\left(\stackrel{\circ}{G}^{i j k l}\left(\xi \partial_{k}\left(d_{V} U_{i j}\right)-\xi_{, k} d_{V} U_{i j}\right)\right)=0
$$

(здесь $\stackrel{\circ}{\nabla}_{k}=\partial_{k}$ в декартовых координатах) в силу того, что подынтегральное выражение в правой части равно нулю, поскольку $\partial_{k} \partial_{l} \xi=0$ и $G^{i j k l} \partial_{k} \partial_{l} U_{i j}=\partial^{i} \partial^{j} U_{i j}-\stackrel{\Delta}{\Delta}=0$. Итак, поверхностный интеграл для бустов не имеет расходимостей.

Аналогично возможная расходимость момента импульса имеет вид $2 \oint \xi^{m} \delta_{k m} d_{V} V^{k l} d^{2} S_{l}$, где остается только четная составляющая ведущей части импульса $\pi^{i j}$ в силу того, что относительно пространственных вращений $\xi^{k}$ является нечетной. Снова, поскольку в ведущем порядке условия связи выполнены, этот интеграл равен нулю, как интеграл от точной 2-формы по замкнутой двумерной поверхности, или, что эквивалентно,

$$
2 \oint \xi_{k} d_{V} V^{k l} d^{2} S_{l}=2 \int d^{3} x \partial_{l}\left(\xi_{k} d_{V} V^{k l}\right)=0 .
$$

Это равенство можно получить, используя соотношения $\partial_{l} \xi_{k}+\partial_{k} \xi_{l}=0$ и $\partial_{l} V^{k l}=0$.

Подчеркнем, что для нашего доказательства нужно только, чтобы нечетная часть поля $\bar{h}_{i j}$ и четная часть импульса $\bar{\pi}^{i j}$ в ведущем порядке удовлетворяли условиям связи. В нашем случае, когда эти части задаются несобственными калибровочными преобразованиями, это 
требование выполнено автоматически и потому по сути не является независимым. Однако для альтернативных граничных условий, приведенных в работе [18], такое условие не выполняется автоматически и должно быть наложено дополнительно. (Тот факт, что условие связи выполняется асимптотически, в любом случае используется для доказательства конечности симплектической формы для каждого из наборов граничных условий.)

6.2. Импульс и момент импульса. Установив, что поверхностный интеграл конечен на бесконечности, мы должны теперь проверить, что он является точным ("интегрируемым") в пространстве полей; иначе говоря, его можно записать в виде внешней производной $-d_{V} \mathcal{B}_{\xi}^{\text {grav }}$ поверхностного члена $\mathcal{B}_{\xi}^{\text {grav }}$, который мы потом добавим к объемному интегралу $\int d^{3} x\left(\xi \mathcal{H}+\xi^{i} \mathcal{H}_{i}\right)$.

Анализ бустов достаточно громоздок, и проще всего провести его в сферических координатах. Поверхностные же члены момента импульса и супертрансляций, напротив, определены уже непосредственно в декартовых координатах. Начнем с них, а бусты рассмотрим после того, как перепишем соответствующие формулы в сферических координатах.

Момент импульса. Для момента импульса $\left(\xi=0, \xi^{m}=b_{k}^{m} x^{k}=O(r)\right)$ вклад в упомянутый выше поверхностный член порядка $O(1)$ с учетом равенства $\xi^{l} d S_{l}=0$ равен

$$
-2 \oint \xi^{m} \delta_{m k} d_{V} \Pi^{(3) k l} d S_{l}-2 \oint \xi^{m}\left(h_{m k}^{(1)} d_{V} \Pi^{(2) k l}+\Pi^{(2) k l} d_{V} h_{m k}^{(1)}\right) d^{2} S_{l},
$$

где $h_{m k}^{(1)}$ - компонента поля $h_{i j}$ порядка $O\left(r^{-1}\right)$,

$$
h_{i j}=h_{m k}^{(1)}+O\left(r^{-2}\right), \quad h_{m k}^{(1)}=\frac{\bar{h}_{m k}(\mathbf{n})}{r} .
$$

При этом $\Pi^{(2) k l}$ и $\Pi^{(3) k l}$ - это компоненты импульса $\pi^{k l}$ порядков $O\left(r^{-2}\right)$ и $O\left(r^{-3}\right)$ соответственно (в декартовых координатах),

$$
\pi^{k l}=\Pi^{(2) k l}+\Pi^{(3) k l}+O\left(r^{-4}\right), \quad \Pi^{(2) k l}=\frac{\bar{\pi}^{k l}(\mathbf{n})}{r^{2}}, \quad \Pi^{(3) k l}=\frac{\bar{\Pi}^{(3) k l}(\mathbf{n})}{r^{3}} .
$$

Очевидно, что поверхностный член в пространстве полей является точным:

$$
d_{V}\left[-2 \oint \xi^{m}\left(\delta_{m k} \Pi^{(3) k l}+h_{m k}^{(1)} \Pi^{(2) k l}\right) d S_{l}\right] .
$$

Поэтому, подбирая постоянную интегрирования так, чтобы пространство Минковского имело нулевой момент импульса, находим, что поверхностный член, который нужно добавить к интегралу $\int d^{3} x \xi^{i} \mathcal{H}_{i}$ для вращений, имеет вид

$$
\frac{1}{2} b_{m n} M^{m n}, \quad M^{m n}=4 \oint x^{[n}\left(\Pi^{(3) m] l}+\delta^{m] p} h_{p k}^{(1)} \Pi^{(2) k l}\right) d S_{l}
$$

(с антисимметризацией веса 1).

Cупертрансляиии. Теперь рассмотрим супертрансляции - их порождают $\xi=T$ и $\xi_{i}=$ $=\partial_{i}(\widetilde{W}), \widetilde{W}=r W$. Вклад порядка $O(1)$ в поверхностный интеграл на бесконечности имеет вид

$$
-\oint \dot{G}^{i j k l} T\left(d_{V} h_{i j}^{(1)}\right)_{, k} d^{2} S_{l}-\oint\left(2 \xi_{k} d_{V} \Pi^{(2) k l}\right) d^{2} S_{l}
$$

и, очевидно, является интегрируемым в силу линейности по производной $d_{V}$ от полей. Мы опустили член $\oint \stackrel{G}{G}^{i j k l}\left(T_{, k} d_{V} h_{i j}^{(1)}\right) d^{2} S_{l}$, равный нулю в силу того, что $T$ зависит только от 
угловых переменных и, следовательно, $T_{, k} \sim e_{k}^{A}$, в то время как $d^{2} S_{l} \sim n_{l}$, в результате в данном выражении присутствует только $d_{V} h_{r A}^{(1)}$. Но при этом $h_{r A}^{(1)}=0$.

Оказывается, что части полей $h_{i j}^{(1)}$ и $\Pi^{(2) k l}$ с несобственной калибровкой не вносят вклад в интеграл, поэтому поверхностный член, добавляемый к $\int d^{3} x\left(T \mathcal{H}+\partial^{i}(\widetilde{W}) \mathcal{H}_{i}\right)$, сводится к

$$
\mathcal{B}_{\{T, W\}}^{\text {grav }}=\oint \dot{G}^{i j k l} T\left(\left(h_{i j}^{(1)}\right)^{\text {even }}\right)_{, k} d^{2} S_{l}+\oint\left(2 \partial_{k}(\widetilde{W})\left(\Pi^{(2) k l}\right)^{\text {odd }}\right) d^{2} S_{l}
$$

(где $\mathcal{B}_{\{T, W\}}^{\text {grav }}=0$ при $h_{i j}=\pi^{i j}=0$ ). Отсюда следует, что в поверхностном интеграле присутствуют только $T^{\text {even }}$ и $W^{\text {odd }}$.

То, что часть поля $h_{i j}^{(1)}$ с несобственной калибровкой действительно исчезает из поверхностного интеграла, вытекает из того, что $d^{2} S_{l} \sim n_{l}$ и $\stackrel{G}{ }^{i j k l} n_{l} U_{i j, k}=0$ в силу вида (3.20) функции $U_{i j}$ и тождества $\stackrel{\circ}{G}^{i j k l} n_{l}\left(n_{k} e_{i}^{A} e_{j}^{B}+n_{i} e_{k}^{A} e_{j}^{B}+n_{j} e_{i}^{A} e_{k}^{B}\right)=0$. Часть в $\Pi^{(2) k l}$ с несобственной калибровкой исчезает в силу тождества (3.18) и соотношения $\partial_{k}(r W)=n_{k} W+e_{k}^{A} \partial_{A} W$, а также того, что интеграл $\oint \partial_{A} V^{A} d^{2} x$ по сфере от полной производной равен нулю.

Таким образом, генератор супертрансляций задается как

$$
Q_{\{T, W\}}=\int d^{3} x\left(T \mathcal{H}+\partial^{i}(\widetilde{W}) \mathcal{H}_{i}\right)+\mathcal{B}_{\{T, W\}}^{\text {grav }} .
$$

Когда выполнено условие связи, имеем $Q_{\{T, W\}} \approx \mathcal{B}_{\{T, W\}}^{\text {grav }}$; данное выражение равно нулю, если $T$ - четная, а $W$ - нечетная функции. Это означает, что преобразования, порождаемые компонентами $T^{\text {odd }}$ и $W^{\text {even }}$, являются собственными калибровочными преобразованиями, не изменяющими физическое состояние системы. Напротив, преобразования, порождаемые компонентами $T^{\text {even }}$ и $W^{\text {odd }}$, имеют в общем случае ненулевые заряды и представляют собой настоящие несобственные калибровочные преобразования. При $T=1$ и $W=0$ восстанавливается энергия Арновитта-Дезера-Мизнера [1, 25].

Можно упростить выражение для заряда, ассоциированного с супертрансляцией $T$ в направлении, нормальном к гиперповерхностям постоянного времени, используя соотношение $\left(h_{i j}^{(1)}\right)^{\text {even }}=r^{-1} n_{i} n_{j} \bar{h}_{r r}+r e_{i}^{A} e_{j}^{B} \bar{h}_{A B}$, где $\bar{h}_{r r}$ и $\bar{h}_{A B}$ зависят только от углов. Член $\bar{h}_{A B}$ исчезает точно так же, как член $U_{i j}$, и остается только $\bar{h}_{r r}$. Это дает

$$
\mathcal{B}_{\{T, 0\}}^{\text {grav }}=2 \oint d^{2} x \sqrt{\bar{\gamma}} T \bar{h}_{r r} .
$$

Функции хода и сдвига. Функции хода и сдвига $N$ и $N^{k}$, которые являются множителями Лагранжа для условий связи (первого рода), необходимо выбирать так, чтобы граничные условия сохранялись в ходе динамической эволюции. Это означает, что данные функции можно выбрать для параметризации общей асимптотической симметрии. Обычно полагают

$$
N=1+O\left(r^{-1}\right), \quad N^{r}=O\left(r^{-1}\right), \quad N^{A}=O\left(r^{-2}\right) .
$$

Это соответствует сечениям гиперповерхностями, которые асимптотически становятся параллельными гиперплоскостями. Наложение таких граничных условий на функции хода и сдвига приводит к тому, что нужно добавить к действию энергию Арновитта-Дезера-Мизнера

$$
B_{\infty}=\mathcal{B}_{\{1,0\}}^{\text {grav }}=2 \oint d^{2} x \sqrt{\bar{\gamma}} \bar{h}_{r r} .
$$

6.3. Сферические координаты. Чтобы проанализировать интегрируемость зарядов буста, перейдем к сферическим координатам.

Напомним, что сопряженные импульсы характеризуются плотностью веса один. Поэтому в сферических координатах приведенные выше граничные условия для динамических 
переменных имеют вид

$$
\begin{aligned}
& g_{r r}=1+\frac{1}{r} \bar{h}_{r r}+\frac{1}{r^{2}} h_{r r}^{(2)}+o\left(r^{-2}\right), \quad \pi^{r r}=\bar{\pi}^{r r}+\frac{1}{r} \pi^{(2) r r}+o\left(r^{-1}\right), \\
& g_{r A}=\bar{\lambda}_{A}+\frac{1}{r} h_{r A}^{(2)}+o\left(r^{-1}\right) \quad\left(\bar{\lambda}_{A}=0\right), \quad \pi^{r A}=\frac{1}{r} \bar{\pi}^{r A}+\frac{1}{r^{2}} \pi^{(2) r A}+o\left(r^{-2}\right), \\
& g_{A B}=r^{2} \bar{\gamma}_{A B}+r \bar{h}_{A B}+h_{A B}^{(2)}+o(1), \quad \pi^{A B}=\frac{1}{r^{2}} \bar{\pi}^{A B}+\frac{1}{r^{3}} \pi^{(2) A B}+o\left(r^{-3}\right) .
\end{aligned}
$$

Здесь мы оставили член $\bar{h}_{r A} \equiv \bar{\lambda}_{A}$ порядка единицы в $g_{r A}$, чтобы показать, что интегрируемость зарядов буста действительно гарантирована, если наложить условие $\bar{\lambda}_{A}=0$. На самом деле этот член отсутствует. Заметим, что мы используем здесь обозначения $\pi^{(2) r r}, \pi^{(2) r A}$, $\pi^{(2) A B}$ (см. вторые слагаемые в разложениях импульса) для той величины, которая ранее в декартовых координатах обозначалась через $\Pi^{(3) i j}$ (слагаемые порядка $r^{-3}$ ).

Как мы видели выше, ведущие порядки динамических переменных подчиняются двум типам дополнительных условий:

(i) условиям четности;

(ii) условиям связи.

Обобщенные условия четности. При антиподальном отображении $x^{A} \rightarrow-x^{A}$ указанные выше условия четности, включающие в себя несобственный калибровочный твист, таковы:

$$
\begin{gathered}
\bar{h}_{r r} \text { - четная величина, } \quad \bar{\lambda}_{A}-\text { нечетная величина, } \\
\bar{\pi}^{r r}=\left(\bar{\pi}^{r r}\right)^{\text {odd }}-\sqrt{\bar{\gamma}} \bar{\Delta} V, \quad \bar{\pi}^{r A}=\left(\pi^{r A}\right)^{\text {even }}-\sqrt{\bar{\gamma}} \bar{D}^{A} V, \\
\bar{\pi}^{A B}=\left(\bar{\pi}^{A B}\right)^{\text {odd }}+\sqrt{\bar{\gamma}}\left(\bar{D}^{A} \bar{D}^{B} V-\bar{\gamma}^{A B} \bar{\Delta} V\right), \\
\bar{h}_{A B}=\left(\bar{h}_{A B}\right)^{\text {even }}+2\left(\bar{D}_{A} \bar{D}_{B} U+U \bar{\gamma}_{A B}\right),
\end{gathered}
$$

где $V$ - четная, а $U-$ нечетная функции (и $\left.\bar{\Delta} \equiv \bar{D}_{C} \bar{D}^{C}\right)$. Из этих условий вытекает точное соотношение

$$
\bar{\pi}^{r r}-\bar{\pi}_{A}^{A}-\text { нечетная величина. }
$$

Переменные $\bar{h}_{r r}$ и $\bar{\pi}^{r r}-\bar{\pi}_{A}^{A}$ играют, таким образом, особую роль: они инвариантны относительно несобственных калибровочных преобразований, т.е. супертрансляций.

Условия связи. Чтобы записать условия связи в полярных координатах, полезно ввести $(1+2)$-радиальное расщепление трехмерной метрики $g_{i j}$ :

$$
\begin{gathered}
g_{r r}=\lambda^{2}+\gamma_{A B} \lambda^{A} \lambda^{B}, \quad g_{r A}=\gamma_{A B} \lambda^{B}, \quad g_{A B}=\gamma_{A B}, \\
\lambda=1+r^{-1} \bar{\lambda}+r^{-2} \lambda^{(2)}+o\left(r^{-2}\right), \quad \lambda^{A}=r^{-2} \bar{\lambda}^{A}+r^{-3} \lambda^{(2) A}+o\left(r^{-3}\right), \\
\gamma_{A B}=r^{2} \bar{\gamma}_{A B}+r \bar{h}_{A B}+h_{A B}^{(2)}+o(1) .
\end{gathered}
$$

В соответствии с нашими предыдущими соглашениями для величин с чертой сверху мы обозначаем через $D_{A}$ и $\bar{D}_{A}$ ковариантные производные, связанные с $\gamma_{A B}$ и $\bar{\gamma}_{A B}$. Индексы $A, B, \ldots$ полей во всем объеме понижаются и повышаются с помощью метрики $\gamma_{A B}$ и обратной к ней $\gamma^{A B}$, а те же индексы асимптотических полей - с помощью $\bar{\gamma}_{A B}$ и обратной к ней $\bar{\gamma}^{A B}$. Имеем следующий словарь:

$$
\bar{\lambda}=\frac{1}{2} \bar{h}_{r r}, \quad \bar{\lambda}^{A}=\bar{\gamma}^{A B} \bar{h}_{r B} \equiv \bar{\gamma}^{A B} \bar{\lambda}_{B} .
$$


Внешняя кривизна поверхности при постоянном $r$ задается формулой

$$
K_{A B}=\frac{1}{2 \lambda}\left(-\partial_{r} \gamma_{A B}+D_{A} \lambda_{B}+D_{B} \lambda_{A}\right)
$$

и отличается от кривизны $\stackrel{\circ}{K}_{A B}$ плоского пространства (которая растет как $r$ с ростом $r$ ) на поправку порядка единицы. Разложим величину $K_{B}^{A}$, которая в плоском пространстве ведет себя как $r^{-1}$ :

$$
K_{B}^{A}=-r^{-1} \delta_{B}^{A}+r^{-2} \bar{k}_{B}^{A}+r^{-3} k_{B}^{(2)}{ }_{B}^{A}+o\left(r^{-3}\right),
$$

где первое слагаемое отвечает плоскому пространству. Заметим, что это дает

$$
K_{A B}=-r \bar{\gamma}_{A B}+\left(\bar{k}_{A B}-\bar{h}_{A B}\right)+o(1),
$$

так что $\bar{k}_{A B}$ не является коэффициентом при следующем за ведущим члене в разложении для $K_{A B}$ (он определяется через разложение для $K_{B}^{A}$ ). Прямые вычисления приводят к равенству

$$
\bar{k}_{A B}=\frac{1}{2} \bar{h}_{A B}+\bar{\lambda} \bar{\gamma}_{A B}+\frac{1}{2}\left(\bar{D}_{A} \bar{\lambda}_{B}+\bar{D}_{B} \bar{\lambda}_{A}\right)
$$

из которого следует соотношение $\bar{k}=\bar{h} / 2+2 \bar{\lambda}+\bar{D}_{A} \bar{\lambda}^{A}$. Из (обобщенных) условий четности следует, что

$$
\begin{gathered}
\bar{\lambda}-\text { четная величина, } \\
\bar{k}_{A B}=\left(\bar{k}_{A B}\right)^{\text {even }}+\bar{D}_{A} \bar{D}_{B} U+U \bar{\gamma}_{A B} .
\end{gathered}
$$

Различные полезные формулы для $(2+1)$-разложения кривизны приведены в приложении А. Непосредственные выкладки с использованием этих формул показывают, что условия более быстрого, чем ожидалось, убывания связей представляют собой линейные условия для асимптотических полей, имеющие вид

$$
\bar{D}_{A} \bar{D}_{B} \bar{k}^{A B}-\bar{D}_{A} \bar{D}^{A} \bar{k}=0, \quad \bar{\pi}^{r A}+\bar{D}_{B} \bar{\pi}^{A B}=0, \quad \bar{D}_{A} \bar{D}_{B} \bar{\pi}^{A B}+\bar{\pi}_{A}^{A}=0 .
$$

\section{4. Анализ интегрируемости в сферических координатах.}

Асимптотические преобразования симметрии. Асимптотические симметрии, сохраняющие граничные условия, порождаются векторными полями (5.5)-(5.7), которые в сферических координатах имеют вид

$$
\begin{gathered}
\xi=b r+T+C_{(b)}+O\left(r^{-1}\right), \quad \xi^{A}=Y^{A}+\frac{1}{r}\left(\bar{D}^{A} W+C_{(b)}^{A}\right)+O\left(r^{-2}\right), \\
\xi^{r}=W+O\left(r^{-1}\right), \quad \bar{D}_{A} \bar{D}_{B} b+\bar{\gamma}_{A B} b=0, \quad \mathcal{L}_{Y} \bar{\gamma}_{A B}=0,
\end{gathered}
$$

где $b\left(x^{B}\right)$ и $Y^{A}\left(x^{B}\right)$ описывают бусты и пространственные вращения, а $T\left(x^{B}\right)$ и $W\left(x^{B}\right)-$ не зависящие от полей функции на сфере. Имеем $b_{i} x^{i}=b r$ и $Y^{A}=b^{m n} Y_{m n}^{A} / 2$ в терминах базиса $x_{m} \partial_{n}-x_{n} \partial_{m} \equiv Y_{m n}$.

"Поправочные" члены $C_{(b)}$ и $C_{(b)}^{A}$ линейны относительно бустов, зависят от полей и имеют порядок единицы. Поэтому они являются подчиненными по порядку относительно $r b$. Член $C_{(b)}$ необходимо добавить к бустам для того, чтобы их генератор был корректно определен. Мы запишем этот член как $C_{(b)}=b F$ с функцией $F$, которая в силу требования интегрируемости должна быть равна $-\bar{k}$ (с точностью до несущественного множителя $\bar{\lambda}$, см. ниже). Итак,

$$
C_{(b)}=b F, \quad F=-\bar{k} .
$$

Член $C_{(b)}^{A}$ также задает поправку к бустам, которая необходима для выполнения условия $\bar{h}_{r A}=0$. Непосредственные расчеты с использованием преобразования метрики $g_{i j}$ под дей- 
ствием бустов показывают, что этот член равен

$$
C_{(b)}^{A}=\frac{2 b}{\sqrt{\bar{\gamma}}} \bar{\pi}^{r A}
$$

Применяя $(2+1)$-разложение, получаем, что в полярных координатах член (6.3) имеет вид

$$
\begin{aligned}
\mathcal{K}_{\xi}\left[d_{V} g_{i j}, d_{V} \pi^{i j}\right]= & \oint d^{2} x\left\{-2 \xi^{i} d_{V} \pi_{i}^{r}+\xi^{r} \pi^{i j} d_{V} g_{i j}-2 \sqrt{\gamma} \xi d_{V} K-\right. \\
& \left.-\sqrt{\gamma} \gamma^{B C} d_{V} \gamma_{C A}\left(\xi K_{B}^{A}+\frac{1}{\lambda}\left(\partial_{r} \xi-\lambda^{D} \partial_{D} \xi\right) \delta_{B}^{A}\right)\right\} .
\end{aligned}
$$

Далее это выражение можно преобразовать как

$$
\begin{aligned}
\mathcal{K}_{\xi}\left[d_{V} g_{i j}, d_{V} \pi^{i j}\right] & =r \oint d^{2} x\left\{-2 Y^{A} \bar{\gamma}_{A B} d_{V} \bar{\pi}^{r B}-2 \sqrt{\bar{\gamma}} b d_{V} \bar{k}\right\}+ \\
& +\oint d^{2} x\left\{-2 Y^{A} d_{V}\left(\bar{h}_{A B} \bar{\pi}^{r B}+\bar{\gamma}_{A B} \pi^{(2) r B}+\bar{\lambda}_{A} \bar{\pi}^{r r}\right)-2\left(\bar{D}^{A} W+C_{(b)}^{A}\right) \bar{\gamma}_{A B} d_{V} \bar{\pi}^{r B}-\right. \\
& -2 W d_{V} \bar{\pi}^{r r}-\sqrt{\bar{\gamma}}\left(b \bar{h} d_{V} \bar{k}+2(b F+T) d_{V} \bar{k}+2 b d_{V} k^{(2)}\right)+ \\
& \left.+\sqrt{\bar{\gamma}}\left(b F+T+\bar{\lambda} b+\bar{\lambda}^{D} \partial_{D} b\right) d_{V} \bar{h}-\sqrt{\bar{\gamma}} b \bar{k}^{A B} d_{V} \bar{h}_{A B}\right\}+o\left(r^{0}\right) .
\end{aligned}
$$

Еще одна проверка отсутствия расходимостей. Пропорциональное $r$ выражение в первой строке (6.34) - это указанный выше член, который потенциально может линейно расходиться. С учетом (6.28) можно снова проверить, что коэффициент при нем равен нулю. Действительно, имеем

$$
\oint d^{2} x Y^{A} \bar{\gamma}_{A B} d_{V} \bar{\pi}^{r B}=-\oint d^{2} x Y^{A} \bar{\gamma}_{A B} \bar{D}_{C} d_{V} \bar{\pi}^{B C}=\oint d^{2} x \bar{D}_{(C} Y_{B)} d_{V} \bar{\pi}^{B C}=0
$$

поскольку $Y^{A}$ - вектор Киллинга на сфере. Аналогично, интегрируя по частям и учитывая, что $b$ удовлетворяет уравнению $\bar{D}_{A} \bar{D}_{B} b+\bar{\gamma}_{A B} b=0$, получаем

$$
\oint \sqrt{\bar{\gamma}} b d_{V} \bar{k}=\oint \sqrt{\bar{\gamma}} b d_{V}\left(\bar{k}-\bar{D}_{A} \bar{D}_{B} \bar{k}^{A B}+\bar{D}_{A} \bar{D}^{A} \bar{k}\right)=\oint \sqrt{\bar{\gamma}} b d_{V}(\bar{k}+\bar{k}-2 \bar{k})=0 .
$$

Таким образом, точные условия четности не являются необходимыми для устранения линейной расходимости в $\mathcal{K}_{\xi}\left[d_{V} g_{i j}, d_{V} \pi^{i j}\right]$. Можно допустить твист в условиях четности, задаваемый несобственным калибровочным преобразованием, который автоматически дает решение условий связи в ведущем порядке.

Момент импульса и заряды супертрансляций в полярных координатах. Пропорциональный $Y^{A}$ член в конечной части выражения для $\mathcal{K}_{\xi}\left[d_{V} g_{i j}, d_{V} \pi^{i j}\right]$ приводит к построенному выше угловому моменту

$$
\frac{1}{2} b_{m n} M^{m n}=\oint d^{2} x\left\{Y^{A}\left(4 \bar{k}_{A B} \bar{\pi}^{r B}-4 \bar{\lambda} \bar{\gamma}_{A B} \bar{\pi}^{r B}+2 \bar{\gamma}_{A B} \pi^{(2) r B}\right)\right\},
$$

где мы положили $\bar{\lambda}_{A}=0$, предвосхищая результат анализа бустов. Но член $\bar{\lambda}_{A}$ можно было бы здесь и сохранить, так как он не портит интегрируемость момента импульса. Члены, пропорциональные $T$ и $W$, дают заряды супертрансляции $\mathcal{B}_{\{T, W\}}^{\text {grav }}=\oint d^{2} x\left\{2 W\left(\bar{\pi}^{r r}-\bar{\pi}_{A}^{A}\right)+4 T \sqrt{\bar{\gamma}} \bar{\lambda}\right\}$ (мы учли асимптотику условий связи для импульсов). Это выражение можно упростить:

$$
\mathcal{B}_{\{T, W\}}^{\text {grav }}=\oint d^{2} x\left\{2 W^{\text {odd }}\left(\bar{\pi}^{r r}-\bar{\pi}_{A}^{A}\right)+4 T^{\text {even }} \sqrt{\bar{\gamma}} \bar{\lambda}\right\},
$$

поскольку функция $\bar{\pi}^{r r}-\bar{\pi}_{A}^{A}$ нечетная, а $\bar{\lambda}$ четная. Эти формулы полностью согласуются с полученными выше. 
Бусты. Осталось провести анализ бустов, для которых $\mathcal{K}_{\xi}\left[d_{V} g_{i j}, d_{V} \pi^{i j}\right]$ сводится к

$$
\begin{aligned}
\mathcal{K}_{\xi}\left[d_{V} g_{i j}, d_{V} \pi^{i j}\right]= & \oint d^{2} x\left\{-2 C_{(b)}^{A} \bar{\gamma}_{A B} d_{V} \bar{\pi}^{r B}-\sqrt{\bar{\gamma}}\left(b \bar{h} d_{V} \bar{k}+2 b F d_{V} \bar{k}+2 b d_{V} k^{(2)}\right)+\right. \\
& \left.+\sqrt{\bar{\gamma}}\left(b F+\bar{\lambda} b+\bar{\lambda}^{D} \partial_{D} b\right) d_{V} \bar{h}-\sqrt{\bar{\gamma}} b \bar{k}^{A B} d_{V} \bar{h}_{A B}\right\}+o\left(r^{0}\right) .
\end{aligned}
$$

Можно переписать этот интеграл как

$$
\begin{aligned}
\mathcal{K}_{\xi}\left[d_{V} g_{i j}, d_{V} \pi^{i j}\right] & =d_{V} \oint d^{2} x\left\{-2 \sqrt{\bar{\gamma}} b k^{(2)}-\frac{1}{4} \sqrt{\bar{\gamma}} b\left(\bar{h}^{2}+\bar{h}^{A B} \bar{h}_{A B}\right)-b \frac{2}{\sqrt{\bar{\gamma}}} \bar{\gamma}_{A B} \bar{\pi}^{r A} \bar{\pi}^{r B}\right\}+ \\
& +\oint d^{2} x\left\{-\sqrt{\bar{\gamma}}(2 b F+\bar{h} b) d_{V}\left(2 \bar{\lambda}+\bar{D}_{A} \bar{\lambda}^{A}\right)+\sqrt{\bar{\gamma}}\left(\bar{\lambda}^{C} \partial_{C} b \bar{\gamma}^{A B}-b \bar{D}^{A} \bar{\lambda}^{B}\right) d_{V} \bar{h}_{A B}\right\}+ \\
& +o\left(r^{0}\right) .
\end{aligned}
$$

Выражение во второй строке в (6.39) неинтегрируемо без дополнительных ограничений. Это можно увидеть, если вычислить $d_{V} \mathcal{K}_{\xi}$. Далее заметим, что член $d_{V} \bar{h} d_{V} \bar{\lambda}$ в $d_{V} \mathcal{K}_{\xi}$ имеет вид $b\left(2 d_{V} F+d_{V} \bar{h}\right) d_{V} \bar{\lambda}$ и равен нулю, только если положить $F$ равным $-\bar{h} / 2+a \bar{\lambda}$, где постоянная $a$ произвольна. Это совпадает с $(6.31)$ с точностью до множителя $a^{\prime} \bar{\lambda}$. Однако этот интегрируемый член не играет роли, потому что он дает интеграл $\oint d^{2} x b(\bar{\lambda})^{2}$, равный нулю в силу того, что $b$ - нечетная, а $(\bar{\lambda})^{2}$ - четная величина. Поэтому мы можем выбрать его произвольным образом. Для удобства мы взяли $a^{\prime}=0$. Условие $\bar{\lambda}_{A}=0$ делает выражение в последней строке также интегрируемым. Мы не исследовали подробно общее решение условия точности 1-формы в пространстве полей $\mathcal{K}_{\xi}$. Однако, как отмечено в заключении, условие $\bar{\lambda}_{A}=0$ имеет глубокий смысл. Некоторые дополнительные аргументы приведены в приложении В.

Учитывая все эти условия, находим явное выражение для поверхностного члена $\mathcal{B}_{\xi}$, отвечающего бустам (такого, что $\left.d_{V} \mathcal{B}_{\xi}+\mathcal{K}_{\xi}=0\right)$ :

$$
\mathcal{B}_{\xi}\left[g_{i j}, \pi^{i j}\right]=\oint d^{2} x\left\{b \sqrt{\bar{\gamma}}\left(2 k^{(2)}+\bar{k}^{2}+\bar{k}_{B}^{A} \bar{k}_{A}^{B}-6 \bar{\lambda} \bar{k}\right)+b \frac{2}{\sqrt{\bar{\gamma}}} \bar{\gamma}_{A B} \bar{\pi}^{r A} \bar{\pi}^{r B}\right\} .
$$

Мы вновь выбрали константу интегрирования в $\mathcal{B}_{\xi}$ так, чтобы решение в фоновой метрике Минковского имело нулевой заряд.

\section{7. АЛГЕБРА $\mathrm{BMS}_{4}$. СВЕТОПОДОБНАЯ БЕСКОНЕЧНОСТЬ}

Собирая вместе все полученные выше результаты, мы видим, что асимптотические симметрии являются каноническими преобразованиями, порожденными генератором

$$
P_{\xi}^{\text {grav }}\left[g_{i j}, \pi^{i j}\right]=\int d^{3} x\left(\xi \mathcal{H}+\xi^{i} \mathcal{H}_{i}\right)+\mathcal{B}_{\xi}^{\text {grav }}\left[g_{i j}, \pi^{i j}\right] .
$$

Здесь граничный член задается как сумма уже найденных вкладов:

$$
\begin{aligned}
\mathcal{B}_{\xi}\left[g_{i j}, \pi^{i j}\right]= & \oint d^{2} x\left\{Y^{A}\left(4 \bar{k}_{A B} \bar{\pi}^{r B}-4 \bar{\lambda}_{\bar{\gamma}} \bar{\pi}^{r B}+2 \bar{\gamma}_{A B} \pi^{(2) r B}\right)+2 W\left(\bar{\pi}^{r r}-\bar{\pi}_{A}^{A}\right)+\right. \\
& \left.+4 T \sqrt{\bar{\gamma}} \bar{\lambda}+b \sqrt{\bar{\gamma}}\left(2 k^{(2)}+\bar{k}^{2}+\bar{k}_{B}^{A} \bar{k}_{A}^{B}-6 \bar{\lambda} \bar{k}\right)+b \frac{2}{\sqrt{\bar{\gamma}}} \bar{\gamma}_{A B} \bar{\pi}^{r A} \bar{\pi}^{r B}\right\} .
\end{aligned}
$$

Заметим, что заряд буста и момент импульса включают в себя вклады, квадратичные по асимптотическим полям. Они отсутствуют для точных условий четности [25]. Если сделать преобразование БМС вдали от "нетвистованной" системы координат, то возникают квадратичные вклады. 
Нетрудно найти алгебру

$$
\left\{P_{\xi_{1}}^{\text {grav }}\left[g_{i j}, \pi^{i j}\right], P_{\xi_{2}}^{\text {grav }}\left[g_{i j}, \pi^{i j}\right]\right\}=P_{\widehat{\xi}}^{\text {grav }}\left[g_{i j}, \pi^{i j}\right],
$$

где $\widehat{\xi}$ порождает асимптотическую симметрию со следующими параметрами:

$$
\begin{aligned}
\widehat{Y}^{A} & =Y_{1}^{B} \partial_{B} Y_{2}^{A}+\bar{\gamma}^{A B} b_{1} \partial_{B} b_{2}-(1 \leftrightarrow 2), \\
\widehat{b} & =Y_{1}^{B} \partial_{B} b_{2}-(1 \leftrightarrow 2), \\
\widehat{T} & =Y_{1}^{A} \partial_{A} T_{2}-3 b_{1} W_{2}-\partial_{A} b_{1} \bar{D}^{A} W_{2}-b_{1} \bar{D}_{A} \bar{D}^{A} W_{2}-(1 \leftrightarrow 2), \\
\widehat{W} & =Y_{1}^{A} \partial_{A} W_{2}-b_{1} T_{2}-(1 \leftrightarrow 2) .
\end{aligned}
$$

Поскольку бусты задаются нечетными функциями $b$, мы видим, что в результате преобразований Лоренца $T^{\text {even }}$ смешивается с $W^{\text {odd }}$, a $T^{\text {odd }}-$ c $W^{\text {even }}$. Другими словами, пара $(T, W)$ не образует неприводимого представления группы Лоренца, а распадается на отдельные лоренцевы мультиплеты $\left(T^{\mathrm{even}}, W^{\mathrm{odd}}\right)$ и $\left(T^{\mathrm{odd}}, W^{\mathrm{even}}\right)$. Мультиплет $\left(T^{\mathrm{even}}, W^{\mathrm{odd}}\right)$ порождает физические ("несобственные") калибровочные преобразования и содержит трансляции. Мультиплет $\left(T^{\text {odd }}, W^{\text {even }}\right)$ порождает чистые калибровочные преобразования, которые не изменяют физическое состояние системы. Точные условия четности исключают физические преобразования $\left(T^{\mathrm{even}}, W^{\mathrm{odd}}\right)$, что не является приемлемой фиксацией калибровки.

После отождествления тривиальных преобразований, порожденных четными $W$ и нечетными $T$, полученная алгебра становится алгеброй из работы [18]. С использованием результатов статьи [30] мы показали в [18], что данная алгебра является алгеброй $\mathrm{BMS}_{4}$ в необычной параметризации. Это было сделано путем интегрирования уравнений движения для параметров симметрии всюду вплоть до светоподобной бесконечности по аналогии с работами [3-5, 13-15]. Было обнаружено, что нечетные $W$ и четные $T$ складываются в произвольную функцию угловых переменных, определяющую супертрансляции в параметризации светоподобной бесконечности. Это позволяет сделать вывод, что алгебра симметрий в пространственной бесконечности - это та же алгебра $\mathrm{BMS}_{4}$, что и алгебра $\mathrm{BMS}_{4}$, которая возникает в светоподобной бесконечности. Заметим также, что из нашего анализа вытекают условия соответствия между значениями полей на критических сферах прошлого и будущего (т.е. на границе прошлого для будущей светоподобной бесконечности и границе будущего для прошлой светоподобной бесконечности). Эти условия были сформулированы в [29]. За подробностями мы отсылаем читателя к работам [18, 20, 30].

\section{8. ЗАКЛЮЧЕНИЕ}

Мы привели обзор наших результатов по гамильтоновой формулировке теории гравитации Эйнштейна в контексте асимптотически плоского пространства-времени [18-20]. Предложенные нами граничные условия на пространственной бесконечности приводят к непротиворечивой динамике, инвариантной относительно группы БМС, которая обладает нетривиальным действием.

Наши граничные условия характеризуются двумя ключевыми свойствами.

1. Прежде всего они включают в себя условия четности в ведущем порядке разложения полей, отличающиеся от точных условий четности твистом, который представляет собой несобственное калибровочное преобразование. Эти обобщенные условия четности делают симплектическую форму и лоренцевы заряды конечными, что и послужило мотивацией для их введения.

Полезность введения обобщенных условий четности можно объяснить иначе. Для тензора Вейля общих исходных данных, не удовлетворяющих никакому условию четности, по мере

ТРУДЫ МАТЕМАТИЧЕСКОГО ИНСТИТУТА ИМ. В.А. СТЕКЛОВА, 2020, т. 309 
приближения к светоподобной бесконечности возникают логарифмические сингулярности, если только не наложить точные условия четности на компоненты тензора Вейля $[2,23,30]$. Эти точные условия четности на пространственной бесконечности можно сформулировать как условия того, что электрическая и магнитная компоненты тензора Вейля, которые строго инвариантны при собственных и несобственных калибровочных преобразованиях, являются строго четными в декартовых координатах. Вместе с условиями четности, согласно которым БМС-инвариант $\bar{\lambda}$ (связанный с массой) должен быть четным, а сопряженный ему БМС-инвариант $\bar{\pi}^{r r}-\bar{\pi}_{A}^{A}$ (связанный с импульсом) - нечетным, эти точные условия четности для тензора Вейля влекут за собой наши обобщенные условия четности [20]. ${ }^{5}$

Аналогичные свойства присутствуют и в электромагнетизме [19], а также возникают для 2-формы калибровочных полей [21]. Отметим, однако, что условия сходимости симплектической формы и отсутствия сингулярностей физических полей не приводят к ограничениям на четность в высших измерениях, по крайней мере в электромагнетизме [22]. Предварительные исследования показывают, что, по-видимому, это также относится к гравитации; остается выяснить, можно ли получить согласованную формулировку теории Эйнштейна в высших измерениях без условий четности.

2. Второй ключевой ингредиент - это соотношение $\bar{h}_{r A}=0$, которое говорит о том, что ожидаемый ведущий порядок смешанных радиально-угловых компонент метрики должен быть на самом деле равен нулю. Это условие достаточно для того, чтобы сделать заряды буста интегрируемыми. Разумность этого условия определяется еще одним его важным следствием. Как мы видели, оно связывает угловую часть $\xi^{A}$ несобственных диффеоморфизмов с их радиальной частью $\xi^{r}$. Именно это приводит к тому, что в пространственной бесконечности возникает группа БМС. Аналогичная связь между $\xi^{r}$ и $\xi^{A}$ играет центральную роль и в контексте пространства АдС, где также естественным образом вводится аналог соотношения $\bar{h}_{r A}=0$ (см. $[17,9])$. В приложении В мы приводим дополнительные аргументы в пользу необходимости условия $\bar{h}_{r A}=0$. Во-первых, мы исследуем более подробно интегрируемость выражения (6.39), которое вносит вклад в заряды буста с канонической симплектической структурой, принятой в нашей работе, и показываем, что, хотя возможны и более общие поля с нетривиальными $\bar{h}_{r A}$, физически они эквивалентны полям с условием $\bar{h}_{r A}=0$. Во-вторых, мы исследуем альтернативный подход, предложенный в результате анализа электромагнетизма [19] и калибровочного поля, заданного в виде 2-формы [21]; в рамках этого подхода модификация симплектической структуры связана с поверхностными членами. Мы опять же получаем, что этот вариант физически эквивалентен случаю $\bar{h}_{r A}=0$.

Нетривиальная структура в пространственной бесконечности является результатом того, что существуют физические степени свободы, т.е. степени свободы, инвариантные при "малых" или "собственных" калибровочных преобразованиях, которые при этом не инвариантны при "больших" или "несобственных" калибровочных преобразованиях. Поэтому решающее значение имеет различие между собственными и несобственными калибровочными преобразованиями. Это различие связано с зарядами, которые определяются с помощью стандартной нётеровой процедуры из симметрий действия. Для этого требуется корректно определенное (конечное) действие, которое на самом деле является инвариантным. Заряды тождественно равны нулю (для данного набора граничных условий) в случае собственных калибровочных преобразований или могут быть ненулевыми в случае несобственных калибровочных преобразований. Граничные условия играют существенную роль как в определении симметрий (которые должны оставлять граничные условия и действие инвариантными), так и при фактическом вычислении зарядов.

\footnotetext{
${ }^{5}$ Тот факт, что условия соответствия из работы [29] следуют из обобщенных условий четности, дает дополнительную мотивацию.
} 
Важным вопросом является влияние наших граничных условий на структуру физических состояний в квантовой теории и, в частности, на роль БМС-зарядов в процедуре одевания физических операторов $[8,16]$; эту проблему мы оставляем для дальнейших исследований.

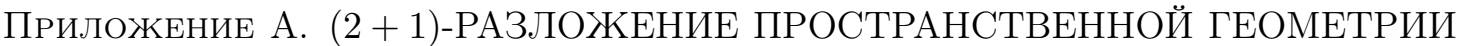

В этом приложении полярные координаты обозначаются через $x^{i}=\left(r, x^{A}\right)$, где $x^{A}$ - координаты на 2-сфере. Мы вводим "ход" $\lambda$ и "сдвиг" $\lambda^{A}$ в соответствии с разделением пространства на слои сферами постоянного радиуса $r$,

$$
\gamma_{A B} \equiv g_{A B}, \quad \lambda_{A} \equiv g_{r A}, \quad \lambda \equiv \frac{1}{\sqrt{g^{r r}}} .
$$

В этих терминах метрика и обратная к ней принимают вид

$$
g_{i j}=\left(\begin{array}{cc}
\lambda^{2}+\lambda_{C} \lambda^{C} & \lambda_{B} \\
\lambda_{A} & \gamma_{A B}
\end{array}\right), \quad g^{i j}=\left(\begin{array}{cc}
1 / \lambda^{2} & -\lambda^{B} / \lambda^{2} \\
-\lambda^{A} / \lambda^{2} & \gamma^{A B}+\lambda^{A} \lambda^{B} / \lambda^{2}
\end{array}\right),
$$

где для поднятия и опускания угловых индексов $A, B, \ldots$ мы используем $\gamma_{A B}$ и обратную к ней $\gamma^{A B}$.

Вводя внешнюю кривизну 2-сфер $K_{A B}$, можно записать символы Кристоффеля:

$$
\begin{aligned}
K_{A B} & =\frac{1}{2 \lambda}\left(-\partial_{r} g_{A B}+D_{A} \lambda_{B}+D_{B} \lambda_{A}\right), \\
\Gamma_{A B}^{r} & =\frac{1}{\lambda} K_{A B}, \\
\Gamma_{B C}^{A} & ={ }^{\gamma} \Gamma_{B C}^{A}-\frac{\lambda^{A}}{\lambda} K_{B C}, \\
\Gamma_{r A}^{r} & =\frac{1}{\lambda}\left(\partial_{A} \lambda+K_{A B} \lambda^{B}\right), \\
\Gamma_{r r}^{r} & =\frac{1}{\lambda} \partial_{r} \lambda+\frac{\lambda^{A}}{\lambda}\left(\partial_{A} \lambda+K_{A B} \lambda^{B}\right), \\
\Gamma_{r B}^{A} & =-\frac{\lambda^{A}}{\lambda}\left(\partial_{B} \lambda+K_{B C} \lambda^{C}\right)+D_{B} \lambda^{A}-\lambda K_{B}^{A}, \\
\Gamma_{r r}^{A} & =-\lambda\left(\gamma^{A B}+\frac{\lambda^{A} \lambda^{B}}{\lambda^{2}}\right)\left(\partial_{B} \lambda+K_{B C} \lambda^{C}\right)-\lambda^{C}\left(D^{A} \lambda_{C}-\lambda K_{C}^{A}\right)-\frac{\lambda^{A}}{\lambda} \partial_{r} \lambda+\gamma^{A B} \partial_{r} \lambda_{B},
\end{aligned}
$$

где $D_{A}$ - ковариантная производная, ассоциированная с $\gamma_{A B}$.

Тензор Риччи задается как

$$
\begin{aligned}
{ }^{(3)} R_{A B} & =\frac{1}{\lambda} \partial_{r} K_{A B}+2 K_{A C} K_{B}^{C}-K K_{A B}-\frac{1}{\lambda} D_{A} D_{B} \lambda+{ }^{\gamma} R_{A B}-\frac{1}{\lambda} \mathcal{L}_{\lambda} K_{A B}, \\
{ }^{(3)} R_{r A} & =\lambda\left(\partial_{A} K-D_{B} K_{A}^{B}\right)+{ }^{(3)} R_{A B} \lambda^{B}, \\
{ }^{(3)} R_{r r} & =\lambda\left(\partial_{r} K-\lambda^{A} \partial_{A} K\right)-\lambda^{2} K_{B}^{A} K_{A}^{B}-\lambda D_{A} D^{A} \lambda-{ }^{(3)} R_{A B} \lambda^{A} \lambda^{B}+2^{(3)} R_{r B} \lambda^{B},
\end{aligned}
$$

а скалярная кривизна имеет вид

$$
{ }^{(3)} R=\frac{2}{\lambda}\left(\partial_{r} K-\lambda^{A} \partial_{A} K\right)+{ }^{\gamma} R-K_{B}^{A} K_{A}^{B}-K^{2}-\frac{2}{\lambda} D_{A} D^{A} \lambda .
$$




\section{ПРИЛОЖЕНИЕ Б. АСИМПТОТИЧЕСКОЕ ПРЕОБРАЗОВАНИЕ ПОЛЕЙ}

Действие асимптотических симметрий на асимптотические поля можно найти, используя вариацию метрики и ее импульса:

$$
\begin{aligned}
\delta_{\xi} \bar{k}_{A B}= & \mathcal{L}_{Y} \bar{k}_{A B}+\bar{D}_{A} \bar{D}_{B} W+W \bar{\gamma}_{A B}+ \\
& +\frac{b}{\sqrt{\bar{\gamma}}}\left(\bar{\pi}_{A B}-\bar{\gamma}_{A B} \bar{\pi}_{C}^{C}\right)+\frac{1}{\sqrt{\bar{\gamma}}} \bar{D}_{A}\left(b \bar{\pi}^{r C} \bar{\gamma}_{C B}\right)+\frac{1}{\sqrt{\bar{\gamma}}} \bar{D}_{B}\left(b \bar{\pi}^{r C} \bar{\gamma}_{C A}\right), \\
\delta_{\xi} \bar{\lambda}= & \frac{b}{4 \sqrt{\bar{\gamma}}} \bar{p}+Y^{C} \partial_{C} \bar{\lambda} \\
\delta_{\xi}\left(\bar{\pi}^{r r}-\bar{\pi}_{A}^{A}\right)= & \mathcal{L}_{Y}\left(\bar{\pi}^{r r}-\bar{\pi}_{A}^{A}\right)+\sqrt{\bar{\gamma}}\left(2 b \bar{D}_{C} \bar{D}^{C} \bar{\lambda}+2 \bar{D}^{C} b \partial_{C} \bar{\lambda}+6 b \bar{\lambda}\right), \\
\delta_{\xi} \bar{\pi}^{r A}= & \mathcal{L}_{Y} \bar{\pi}^{r A}+\sqrt{\bar{\gamma}}\left(\bar{D}_{B}\left(b \bar{k}^{B A}\right)+\bar{D}^{A} b \bar{k}-\bar{D}^{A} T\right), \\
\delta_{\xi} \bar{\pi}^{A B}= & \mathcal{L}_{Y} \bar{\pi}^{A B}+\sqrt{\bar{\gamma}}\left(\bar{D}^{A} \bar{D}^{B} T-\bar{\gamma}^{A B} \bar{D}_{C} \bar{D}^{C} T\right)+3 b \sqrt{\bar{\gamma}}\left(\bar{k}^{A B}-\bar{\gamma}^{A B} \bar{k}\right)+ \\
& +\sqrt{\bar{\gamma}} b\left(\bar{\gamma}^{A B} \bar{D}_{C} \bar{D}^{C} \bar{k}^{A}+\bar{D}_{C} \bar{D}^{C} \bar{k}^{A B}-\bar{D}_{C} \bar{D}^{A} \bar{k}^{C B}-\bar{D}_{C} \bar{D}^{B} \bar{k}^{C A}\right)+ \\
& +\sqrt{\bar{\gamma}}\left(-\bar{D}^{A} b \bar{D}^{B} \bar{k}^{C}-\bar{D}^{B} b \bar{D}^{A} \bar{k}^{A}+\bar{\gamma}^{A B} \bar{D}_{C} b \bar{D}^{C} \bar{k}+2 \bar{\gamma}^{A B} \bar{D}^{D} \bar{k}_{D}^{C} \partial_{C} b-\right. \\
& \left.-\bar{D}^{A} \bar{k}^{B C} \partial_{C} b-\bar{D}^{B} \bar{k}^{A C} \partial_{C} b+\bar{D}^{C} \bar{k}^{A B} \partial_{C} b\right) .
\end{aligned}
$$

Из этих правил преобразования можно получить, что вариации функций $(U)^{\text {odd }}$ и $(V)^{\text {even }}$ имеют следующий вид:

$$
\begin{aligned}
& \delta_{\xi}(U)^{\text {odd }}=Y^{C} \partial_{C}(U)^{\text {odd }}-b(V)^{\text {even }}+(W)^{\text {odd }} \\
& \delta_{\xi}(V)^{\text {even }}=Y^{C} \partial_{C}(V)^{\text {even }}-3 b(U)^{\text {odd }}-\partial_{A} b \bar{D}^{A}(U)^{\text {odd }}-b \bar{D}_{A} \bar{D}^{A}(U)^{\text {odd }}+(T)^{\text {even }} .
\end{aligned}
$$

Эти преобразования содержат неоднородную составляющую $\left((W)^{\text {odd }}\right.$ для $U$ и $(T)^{\text {even }}$ для $\left.V\right)$. Отсюда следует, что если исходить из конфигурации, удовлетворяющей нетвистованным условиям четности, то это в общем случае порождает ненулевой твист, который в точности имеет предписанный вид, за исключением случая, когда преобразование ограничивается алгеброй Пуанкаре: в этом случае твист остается нулевым. Инвариантность граничных условий для расширенного множества преобразований в этом смысле получается напрямую.

\section{ПРИЛОЖЕНИЕ В. ДОПОЛНИТЕЛЬНЫЙ АНАЛИЗ УСЛОВИЙ ИНТЕГРИРУЕМОСТИ ЗАРЯДОВ БУСТА}

В.1. Дальнейшее исследование интегрируемости выражения (6.39). Далее мы анализируем условия, вытекающие из требования, что выражение (6.39) должно быть интегрируемым. В рамках принятой в работе симплектической структуры мы утверждаем, что на самом деле переменные $\bar{\lambda}^{A}$ не должны быть нулевыми, но можно сделать их равными нулю с помощью переопределения координат, которое не влияет на заряды (собственное калибровочное преобразование).

Чтобы второе слагаемое подынтегральной функции во второй строке равенства (6.39) было интегрируемым, необходимо выполнение условия

$$
\oint d^{2} x \sqrt{\bar{\gamma}}\left(d_{V} \bar{\lambda}^{C} \partial_{C} b \bar{\gamma}^{A B}-b \bar{D}^{A}\left(d_{V} \bar{\lambda}^{B}\right)\right) d_{V} \bar{h}_{A B}=0 .
$$


Четная часть поля $\bar{h}_{A B}$ произвольна, а это приводит к тому, что четная часть переменной $\bar{\lambda}^{A}$ должна быть равна нулю ${ }^{6}$. Нечетная часть поля $\bar{h}_{A B}$ должна иметь вид (6.16), откуда с помощью интегрирования по частям, используя соотношение $\bar{D}_{A} \bar{D}_{B} b+\bar{\gamma}_{A B} b=0$, получаем

$$
\oint d^{2} x \sqrt{\bar{\gamma}}\left(-\bar{D}_{A} b \bar{D}^{A} d_{V} \Lambda-b \bar{\Delta} d_{V} \Lambda-4 b d_{V} \Lambda\right) d_{V} \bar{U}=0,
$$

где

$$
\Lambda \equiv \bar{D}_{A}\left(\bar{\lambda}^{A}\right)^{\text {odd }}
$$

Чтобы равенство (В.2) выполнялось для функций $\bar{U}$ без ограничений (нечетных), надо потребовать выполнения равенства

$$
-\bar{D}_{A} b \bar{D}^{A} d_{V} \Lambda-b \bar{\Delta} d_{V} \Lambda-4 b d_{V} \Lambda=0 .
$$

Взяв в качестве $b$ произвольный буст, легко находим, что $d_{V} \Lambda$ должно быть равно нулю. Отсюда следует, что $\Lambda$ не должна зависеть от полей. Самый простой выбор - это $\Lambda=0$, что дает

$$
\bar{\lambda}^{A}=\left(\bar{\lambda}^{A}\right)^{\text {odd }}, \quad \bar{D}_{A}\left(\bar{\lambda}^{A}\right)^{\text {odd }}=0 .
$$

Можно рассматривать также иные способы выбора с $\bar{D}_{A}\left(\bar{\lambda}^{A}\right)^{\text {odd }} \neq 0$, но мы не исследовали их состоятельность.

Теперь рассмотрим следствия из условий (В.5). Когда они выполняются, второе слагаемое в подынтегральном выражении во второй строке равенства (6.39) не просто интегрируемо, а на самом деле равно нулю, что можно увидеть с помощью того же интегрирования по частям. Переменные $\bar{\lambda}^{A}$ не вносят вклад в заряды. Фактически можно положить их равными нулю с помощью собственного калибровочного преобразования. Действительно, если мы переопределим углы как

$$
x^{\prime A}=x^{A}+\frac{1}{r} \bar{\zeta}^{A}, \quad \bar{\zeta}^{A}-\text { нечетная величина, } \quad D_{A} \bar{\zeta}^{A}=0,
$$

то получим

$$
\bar{\lambda}^{A} \rightarrow \bar{\lambda}^{A}+\bar{\zeta}^{A}
$$

Таким образом, сохраняется вид условия (В.5) для $\bar{\lambda}^{A}$. Если положить $\bar{\zeta}^{A}=-\bar{\lambda}^{A}$, то новая $\bar{\lambda}^{A}$ равна нулю. Такое преобразование разрешено, потому что его заряд

$$
\oint \bar{\zeta}^{A} \bar{\gamma}_{A B} \bar{\pi}^{r B}
$$

равен нулю. В самом деле, если $\bar{\zeta}^{A}$ нечетная, то только нечетная часть поля $\bar{\pi}^{r B}$ вносит вклад в интеграл, который принимает вид

$$
\oint d^{2} x \bar{\zeta}^{A} \bar{\gamma}_{A B} \bar{\pi}^{r B}=-\oint d^{2} x \sqrt{\bar{\gamma}} \bar{\zeta}^{A} \bar{D}_{A} V=\oint d^{2} x \sqrt{\bar{\gamma}} \bar{D}_{A} \bar{\zeta}^{A} V=0 .
$$

В.2. Модификация симплектической структуры с помощью поверхностного члена. Существует еще один способ гарантировать существование корректно определенных зарядов для бустов. Он состоит в модификации симплектической структуры с помощью поверхностного члена. При анализе асимптотических симметрий теории электромагнетизма [19]

\footnotetext{
${ }^{6}$ Точнее, $d_{V}\left(\bar{\lambda}^{A}\right)^{\text {even }}=0$, откуда следует, что $\left(\bar{\lambda}^{A}\right)^{\text {even }}$ не должна зависеть от полей. Не существует не зависящего от полей ненулевого векторного поля, к которому можно было бы приравнять $\left(\bar{\lambda}^{A}\right)^{\text {even }}$ без нарушения условий симметрии, поэтому мы выбираем $\left(\bar{\lambda}^{A}\right)^{\text {even }}=0$.
} 
и калибровочных полей, заданных в виде 2-формы [21], мы столкнулись с подобной проблемой интегрируемости для зарядов буста. В указанных случаях, в которых дополнительные ограничения для асимптотических полей могут привести к исчезновению физически важных симметрий, предлагаемое решение проблемы состояло в добавлении к симплектической структуре граничного члена. Подобный подход также применим и в нашем случае, но, как мы увидим ниже, это снова эквивалентно решению, полученному путем наложения условия $\bar{\lambda}^{A}=0$.

Асимптотические условия, рассматриваемые в этом пункте, представляют собой обобщенные условия четности (3.2)-(3.4) и (3.7)-(3.9) вместе с асимптотическими связями (3.12). Однако мы не требуем выполнения соотношений (3.15) и (3.16).

Сначала мы приводим вид альтернативного решения, затем проверяем, что заряды буста корректно определены, и, наконец, объясняем, почему с физической точки зрения этот случай эквивалентен случаю более простого решения $\bar{\lambda}^{A}=0$.

Действие модифицируется путем введения поверхностного члена

$$
2 \oint d^{2} x \bar{\pi}^{r A} \bar{\gamma}_{A B} \partial_{t} \bar{\lambda}^{B}
$$

содержащего производную по времени от $\bar{\lambda}^{A}$. Таким образом, действие принимает следующий вид (включающий член энергии, поскольку функция хода стремится к 1 на бесконечности):

$$
\begin{aligned}
& S\left[g_{i j}, \pi^{i j}, N, N^{i}\right]= \int d t\left\{\int d^{3} x\left(\pi^{i j} \partial_{t} g_{i j}-N^{i} \mathcal{H}_{i}^{\text {grav }}-N \mathcal{H}^{\text {grav }}\right)+\right. \\
&\left.+2 \oint d^{2} x\left(\bar{\pi}^{r A} \bar{\gamma}_{A B} \partial_{t} \bar{\lambda}^{B}-\sqrt{\bar{\gamma}} \bar{h}_{r r}\right)\right\} \\
& \mathcal{H}^{\text {grav }}=-\sqrt{g} R+\frac{1}{\sqrt{g}}\left(\pi^{i j} \pi_{i j}-\frac{1}{2} \pi^{2}\right), \quad \mathcal{H}_{i}^{\text {grav }}=-2 \nabla_{j} \pi_{i}^{j}, \\
& N=1+O\left(r^{-1}\right), \quad N^{r}=O\left(r^{-1}\right), \quad N^{A}=O\left(r^{-2}\right) .
\end{aligned}
$$

Вариация поверхностного вклада в кинетический член дает два (очевидно, дополнительных) уравнения движения

$$
\partial_{t} \bar{\pi}^{r A}=0, \quad \partial_{t} \bar{\lambda}^{A}=0
$$

Однако, поскольку эти два уравнения уже вытекают из уравнений движения во всем пространстве, динамика остается неизменной. В результате модификации действия в симплектическую структуру добавляется граничный член:

$$
\Omega=\int d^{3} x d_{V} \pi^{i j} d_{V} g_{i j}+2 \oint d^{2} x d_{V} \bar{\pi}^{r A} \bar{\gamma}_{A B} d_{V} \bar{\lambda}^{B},
$$

и она не имеет стандартную каноническую форму "Дарбу".

Граничные условия сохраняются векторными полями с асимптотическим поведением вида

$$
\begin{gathered}
\xi=r b+F_{(b)}+O\left(r^{-1}\right), \quad \xi^{r}=W+O\left(r^{-1}\right), \quad \xi^{A}=Y^{A}+r^{-1} I^{A}+O\left(r^{-2}\right) \\
F_{(b)}=T-b \bar{k}-\bar{\lambda}^{C} \partial_{C} b, \quad \bar{D}_{A} \bar{D}_{B} b+\bar{\gamma}_{A B} b=0, \quad \bar{D}_{A} Y_{B}+\bar{D}_{B} Y_{A}=0
\end{gathered}
$$

Мы видим, что по сравнению с генераторами алгебры $\mathrm{BMS}_{4}$, рассмотренными в основном тексте настоящей статьи, в следующем за ведущим порядком члене в $\xi$ возникает дополнительный вклад, зависящий от $\bar{\lambda}^{A}$, в то время как такой же член в $\xi^{A}$ теперь полностью произволен. Это происходит из-за того, что не накладывается условие $\bar{h}_{r A}=0$, так что условие $W_{i}=\partial_{i}(r W)$ 
из (5.7) не возникает. Соответствующие преобразования асимптотических полей $\bar{\lambda}^{A}$ и $\bar{\pi}^{r A}$ задаются как

$$
\begin{aligned}
\delta_{\xi} \bar{\lambda}^{A} & =\frac{2 b}{\sqrt{\bar{\gamma}}} \bar{\pi}^{r A}+\mathcal{L}_{Y} \bar{\lambda}^{A}+\bar{D}^{A} W-I^{A}, \\
\delta_{\xi} \bar{\pi}^{r A} & =\mathcal{L}_{Y} \bar{\pi}^{r A}+\sqrt{\bar{\gamma}}\left(\bar{D}_{B}\left(b \bar{k}^{B A}\right)+\bar{D}^{A} b \bar{k}\right)-\sqrt{\bar{\gamma}} \bar{D}^{A}\left(F_{(b)}+b \bar{k}+b \bar{\lambda}+\bar{\lambda}^{B} \partial_{B} b\right) .
\end{aligned}
$$

Действие симметрий на другие асимптотические поля будет приводить к аналогичным модификациям, но для вычисления зарядов нам не понадобятся их явные выражения.

Присутствие в симплектической структуре граничного члена приводит к тому, что стандартное предписание из работы [25] для определения гамильтоновых генераторов следует изменить, чтобы учесть, что $\Omega$ не имеет форму “Дарбу". Самый простой способ сделать такое изменение - вернуться к первоначальному определению гамильтонова генератора: корректно определенный генератор $G_{Q}$ ассоциирован с преобразованием $\delta_{Q}$ тогда и только тогда, когда он удовлетворяет тождеству $-i_{Q} \Omega=d_{V} G_{Q}$. Используя результат, полученный в п. 6.3 , и вариации (В.17) и (В.18), можно показать, что приведенные выше асимптотические симметрии, и в частности бусты, являются гамильтоновыми. Их генераторы задаются как

$$
G_{\xi}=\int d^{3} x\left(\xi \mathcal{H}+\xi^{i} \mathcal{H}_{i}\right)+\mathcal{B}_{\xi},
$$

где граничный член принимает вид

$$
\begin{aligned}
\mathcal{B}_{\xi}= & \oint d^{2} x\left\{2 Y^{A}\left(\bar{h}_{A B} \bar{\pi}^{r B}+\bar{\gamma}_{A B} \pi^{(2) r B}+\bar{\lambda}_{A} \bar{\pi}^{r r}\right)+2 W \bar{\pi}^{r r}+2 \partial_{A} W \bar{\pi}^{r A}+2 \mathcal{L}_{Y} \bar{\lambda}^{A} \bar{\gamma}_{A B} \bar{\pi}^{r B}\right\}- \\
& -\oint d^{2} x\left\{-\frac{2 b}{\sqrt{\bar{\gamma}}} \bar{\pi}^{r A} \bar{\gamma}_{A B} \bar{\pi}^{r B}-2 \sqrt{\bar{\gamma}} b k^{(2)}-4 \sqrt{\bar{\gamma}} T \bar{\lambda}-\frac{b}{4} \sqrt{\bar{\gamma}}\left(\bar{h}^{2}+\bar{h}_{B}^{A} \bar{h}_{A}^{B}\right)+\right. \\
& +6 \sqrt{\bar{\gamma}} b \bar{\lambda} \bar{D}_{B} \bar{\lambda}^{B}+2 \sqrt{\bar{\gamma}} \bar{\lambda}^{D} \partial_{D} b \bar{k}-2 \sqrt{\bar{\gamma}} b \bar{D}^{A} \bar{\lambda}^{B} \bar{k}_{A B}+ \\
& \left.+\sqrt{\bar{\gamma}} b\left(\bar{D}_{A} \bar{\lambda}^{A}\right)^{2}+\sqrt{\bar{\gamma}} b \bar{D}^{A} \bar{\lambda}^{B} \bar{D}_{(A} \bar{\lambda}_{B)}\right\}
\end{aligned}
$$

Итак, канонически реализована полная группа асимптотических симметрий (В.15), (В.16).

Существование указанных генераторов не означает, однако, что алгебра асимптотических симметрий теперь больше, чем $\mathrm{BMS}_{4}$. Можно понять, что параметр $I^{A}$ на самом деле не вносит вклад в граничный член, а это означает, что ассоциированное преобразование является собственным калибровочным преобразованием. Если мы используем это преобразование для фиксации калибровки $\bar{\lambda}^{A}=0$, то действие и генераторы алгебры $\mathrm{BMS}_{4}$ сводятся к рассмотренным в основном тексте. Как мы уже говорили выше, два решения проблемы интегрируемости зарядов буста, представленные в настоящей работе, а именно наложение условия $\bar{\lambda}^{A}=0$ и введение граничного члена в симплектическую структуру, физически эквивалентны. Это дает дополнительные аргументы в пользу предположения $\bar{\lambda}^{A}=0$, принятого в основном тексте.

Благодарности. М. Энно благодарит за гостеприимство Международный институт математики и физики им. Эрвина Шрёдингера, где была написана эта работа.

\section{СПИСОК ЛИТЕРАТУРЫ}

1. Arnowitt R., Deser S., Misner C.W. The dynamics of general relativity // Gravitation: An introduction to current research / Ed. by L. Witten. New York: J. Wiley and Sons, 1962. Ch. 7. P. 227-264; Gen. Relativ. Gravitation. 2008. V. 40, N 9. P. 1997-2027; arXiv: gr-qc/0405109.

2. Ashtekar A., Bombelli L., Reula O. The covariant phase space of asymptotically flat gravitational fields // Mechanics, analysis and geometry: 200 years after Lagrange. Amsterdam: North-Holland, 1991. P. 417-450. 
3. Ashtekar A., Hansen R.O. A unified treatment of null and spatial infinity in general relativity. I: Universal structure, asymptotic symmetries, and conserved quantities at spatial infinity // J. Math. Phys. 1978. V. 19, N 7. P. 1542-1566.

4. Beig R. Integration of Einstein's equations near spatial infinity // Proc. R. Soc. London A. 1984. V. 391. P. 295-304.

5. Beig R., Schmidt B.G. Einstein's equations near spatial infinity // Commun. Math. Phys. 1982. V. 87, N 1. P. $65-80$.

6. Benguria R., Cordero P., Teitelboim C. Aspects of the Hamiltonian dynamics of interacting gravitational gauge and Higgs fields with applications to spherical symmetry // Nucl. Phys. B. 1977. V. 122, N 1. P. 61-99.

7. Bondi H., van der Burg M.G.J., Metzner A.W.K. Gravitational waves in general relativity. VII: Waves from axi-symmetric isolated systems // Proc. R. Soc. London A. 1962. V. 269. P. 21-52.

8. Bousso R., Porrati M. Soft hair as a soft wig // Classical Quantum Gravity. 2017. V. 34, N 20. Pap. 204001; arXiv: 1706.00436 [hep-th].

9. Brown J.D., Henneaux M. Central charges in the canonical realization of asymptotic symmetries: An example from three dimensional gravity // Commun. Math. Phys. 1986. V. 104, N 2. P. 207-226.

10. Bunster C., Portugues R., Cnockaert S., Henneaux M. Monopoles for gravitation and for higher spin fields // Phys. Rev. D. 2006. V. 73, N 10. Pap. 105014; arXiv: hep-th/0601222.

11. Christodoulou D., Klainerman S. The global nonlinear stability of the Minkowski space. Princeton, NJ: Princeton Univ. Press, 1993. (Princeton Math. Ser.; V. 41).

12. Dirac P.A.M. The theory of gravitation in Hamiltonian form // Proc. R. Soc. London A. 1958. V. 246. P. 333-343.

13. Friedrich H. Gravitational fields near space-like and null infinity // J. Geom. Phys. 1998. V. 24, N 2. P. 83-163.

14. Friedrich H., Kánnár J. Bondi-type systems near spacelike infinity and the calculation of the Newman-Penrose constants // J. Math. Phys. 2000. V. 41, N 4. P. 2195-2232; arXiv: gr-qc/9910077.

15. Friedrich H., Kánnár $J$. Calculating asymptotic quantities near space-like and null infinity from Cauchy data // Ann. Phys. 2000. Bd. 9, N 3-5. S. 321-330; arXiv: gr-qc/9911103.

16. Giddings S.B. Gravitational dressing, soft charges, and perturbative gravitational splitting: E-print, 2019. arXiv: 1903.06160 [hep-th].

17. Henneaux M., Teitelboim C. Asymptotically anti-de Sitter spaces // Commun. Math. Phys. 1985. V. 98, N 3. P. 391-424.

18. Henneaux M., Troessaert C. BMS group at spatial infinity: The Hamiltonian (ADM) approach // J. High Energy Phys. 2018. V. 2018, N 03. Pap. 147; arXiv: 1801.03718 [gr-qc].

19. Henneaux M., Troessaert C. Asymptotic symmetries of electromagnetism at spatial infinity // J. High Energy Phys. 2018. V. 2018, N 05. Pap. 137; arXiv: 1803.10194 [hep-th].

20. Henneaux M., Troessaert C. Hamiltonian structure and asymptotic symmetries of the Einstein-Maxwell system at spatial infinity // J. High Energy Phys. 2018. V. 2018, N 07. Pap. 171; arXiv: 1805.11288 [gr-qc].

21. Henneaux M., Troessaert C. Asymptotic structure of a massless scalar field and its dual two-form field at spatial infinity // J. High Energy Phys. 2019. V. 2019, N 05. Pap. 147; arXiv: 1812.07445 [hep-th].

22. Henneaux M., Troessaert C. Asymptotic structure of electromagnetism in higher spacetime dimensions // Phys. Rev. D. 2019. V. 99, N 12. Pap. 125006; arXiv: 1903.04437 [hep-th].

23. Herberthson M., Ludvigsen $M$. A relationship between future and past null infinity // Gen. Relativ. Gravitation. 1992. V. 24, N 11. P. 1185-1193.

24. Misner C.W. The flatter regions of Newman, Unti, and Tamburino's Generalized Schwarzschild Space // J. Math. Phys. 1963. V. 4, N 7. P. 924-937.

25. Regge T., Teitelboim C. Role of surface integrals in the Hamiltonian formulation of general relativity // Ann. Phys. 1974. V. 88, N 1. P. 286-318.

26. Sachs R.K. Gravitational waves in general relativity. VIII: Waves in asymptotically flat space-time // Proc. R. Soc. London A. 1962. V. 270. P. 103-126.

27. Sachs R. Asymptotic symmetries in gravitational theory // Phys. Rev. 1962. V. 128, N 6. P. $2851-2864$.

28. Slavnov A.A. Gauge invariant infrared regularization for non-abelian fields // Phys. Lett. B. 1981. V. 98, N 1-2. P. 57-58.

29. Strominger A. Lectures on the infrared structure of gravity and gauge theory: E-print, 2017. arXiv: 1703.05448 [hep-th].

30. Troessaert C. The BMS4 algebra at spatial infinity // Classical Quantum Gravity. 2018. V. 35, N 7. Pap. 074003 ; arXiv: 1704.06223 [hep-th]. 